\title{
Extragalactic jets with helical magnetic fields: relativistic MHD simulations
}

\author{
R. Keppens ${ }^{1,2,3}$, Z. Meliani ${ }^{2}$, B. van der Holst ${ }^{1, \star}$, and F. Casse ${ }^{4}$ \\ 1 Centre for Plasma Astrophysics, K.U. Leuven (Leuven Mathematical Modeling and Computational Science Center), \\ Celestijnenlaan 200B, 3001 Heverlee, Belgium \\ e-mail: Rony . Keppens@wis . kuleuven . be \\ 2 FOM-Institute for Plasma Physics Rijnhuizen, Nieuwegein, The Netherlands \\ 3 Astronomical Institute, Utrecht University, The Netherlands \\ 4 AstroParticule \& Cosmologie (APC), Université Paris Diderot, 10 rue Alice Domon et Léonie Duquet, \\ 75205 Paris Cedex 13, France \\ e-mail: fcasse@apc.univ-paris7.fr
}

Received 30 November 2007 / Accepted 9 February 2008

\section{ABSTRACT}

\begin{abstract}
Context. Extragalactic jets are judged to harbor dynamically important, organized magnetic fields that presumably aid in the collimation of the relativistic jet flows.

Aims. We here explore the morphology of AGN jets pervaded by helical field and flow topologies by means of grid-adaptive, highresolution numerical simulations. We concentrate on morphological features of the bow shock and the jet beam behind the Mach disk, for various jet Lorentz factors and magnetic field helicities. We investigate the influence of helical magnetic fields on jet beam propagation in an overdense external medium. We adopt a special relativistic magnetohydrodynamic (MHD) viewpoint on the shockdominated AGN jet evolution. Due to the adaptive mesh refinement (AMR), we can concentrate on the long-term evolution of kinetic energy-dominated jets, with beam-averaged Lorentz factor $\Gamma \simeq 7$, as they penetrate denser clouds. These jets have near-equipartition magnetic fields (with the thermal energy) and radially varying $\Gamma(R)$ profiles within the jet radius $R<R_{\mathrm{j}}$ maximally reaching $\Gamma \sim 22$. Methods. We used the AMRVAC code, with a novel hybrid block-based AMR strategy, to compute ideal plasma dynamics in special relativity. We combined this with a robust second-order shock-capturing scheme and a diffusive approach to controlling magnetic monopole errors.

Results. We find that the propagation speed of the bow shock systematically exceeds the value expected from estimates using beamaverage parameters, in accordance with the centrally-peaked $\Gamma(R)$ variation. The helicity of the beam magnetic field is effectively transported down the beam, with compression zones between the diagonal internal cross-shocks showing stronger toroidal field regions. In comparison with equivalent low-relativistic jets $(\Gamma \simeq 1.15)$, which get surrounded by cocoons with vortical backflows filled by mainly toroidal field, the high speed jets only demonstrate localized, strong toroidal field zones within the backflow vortical structures. These structures are ring-like due to our axisymmetry assumption and may further cascade to a smaller scale in 3D. We find evidence of a more poloidal, straight field layer, compressed between jet beam and backflows. This layer decreases the destabilizing influence of the backflow on the jet beam. In all cases, the jet beam contains rich cross-shock patterns, across which part of the kinetic energy gets transfered. For the high-speed reference jet considered here, significant jet deceleration only occurs beyond distances exceeding $O\left(100 R_{\mathrm{j}}\right)$, as the axial flow can reaccelerate downstream to the internal cross shocks. This reacceleration is magnetically aided by field compression across the internal shocks that pinch the flow.
\end{abstract}

Key words. magnetohydrodynamics (MHD) - methods: numerical - ISM: jets and outflows - relativity

\section{Motivation}

Relativistic jets represent extremely energetic phenomena in astrophysics. They are associated with (1) a variety of compact objects, (2) with active galactic nuclei (AGN) carrying energy fluxes of $10^{45}-10^{48} \mathrm{ergs} / \mathrm{s}$ (Celotti et al. 1997; Tavecchio et al. 2000), or (3) with micro-quasar systems (energy flux of $10^{35} \mathrm{ergs} / \mathrm{s}$ ). These high energies are somehow extracted from the inner part of the system, and this energy is transported over long distances by means of a detectable collimated jet. A large amount of this energy gets deposited by the jet in the surrounding medium, as only a small fraction of the jet energy is dissipated in the innermost region (Sambruna et al. 2006a). In many AGNs, the kinetic energy flux is comparable to the bolometric radiative

* Present adress: University of Michigan, Space physics research laboratory, 2455 Hayward Ann Arbor, Michigan 48109-2143, USA. luminosity of the central part (Rawlings \& Saunders 1991; Xu et al. 1999; Sambruna et al. 2006a). This implies that a study of the interaction of the magnetized jet with external medium could help by providing model constraints for jet formation and collimation.

The jet is assumed to be powered by a spinning black hole (Blandford \& Znajek 1977; Begelman et al. 1984) and/or by the surrounding accretion disk corona (Miller \& Stone 2000). The disk jet is launched by general relativistic magnetohydrodynamic (GRMHD) mechanisms and is accelerated to a high Lorentz factor. The observations show that AGN jets propagate in the parsec scale with a Lorentz factor $\Gamma \sim 10-30$ (Kellermann et al. 2004), and in some AGN types (blazar, QSO), the jets are relativistic even on a kpc scale with $\Gamma \sim 5-30$ (Tavecchio et al. 2004). In such objects, the radio to $X$-ray observations cannot be explained by the presence of a unique synchrotron component. 
Scenarios involving synchrotron and inverse Compton scattering of synchrotron photons are able to fit observations, under the assumption that the jet decelerates from the subpc scale to the larger scale (Georgeanopoulos \& Kazanas 2003). This can be achieved if an outer slower layer surrounds the central relativistic jet "spine" (Ghisellini et al. 2005), since continuous electron acceleration acts at the boundary layer between jet sheath and "spine" (Stawarz \& Ostrowski 2002). This kind of jet structure is supported by direct radio observations (Giroletti et al. 2004) and leads to radial jet structure where the Lorentz factor decreases radially, as suggested both in microquasars (Meier 2003) and in AGN (Jester et al. 2006; Dulwich et al. 2007). This transverse jet stratification is also supported by models of jet launch scenarios (Koide et al. 2001; McKinney 2006; Meliani et al. 2006). However, most numerical investigations to date have ignored the radial stratification of the jet velocity and made simplifying assumptions on the magnetic topology. In this paper, we present numerical simulations of the propagation of magnetized, relativistic, radially stratified jets. These are important for understanding the impact of more realistic jet topologies on the surrounding medium and how this in turn helps to deduce properties of jet formation mechanisms. We look in particular at the effects of helical magnetic fields on the relativistic jet propagation in external media.

The magnetic field seems to play a significant role in the jet collimation and contributes to its acceleration ( $\mathrm{Li}$ et al. 1992; Contopoulos 1994; Fendt 1997; Koide et al. 2001; Vlahakis \& Königl 2004; Bogovalov \& Tsinganos 2005; Meliani et al. 2006). There is observational evidence of an intrinsic magnetic field in relativistic jets. In BL-Lac objects, VLBI observations of the polarization of the synchrotron emission, especially the rotation measure, have shown that the magnetic field is systematically tilted from the jet axis (Asada et al. 2002; Gabuzda et al. 2004), which indicates the presence of helical magnetic fields.

Significant progress has been made in numerical modeling of relativistic jet propagation, especially for relativistic hydrodynamic models (Hardee et al. 2005; Perucho et al. 2006). Relativistic MHD studies of jet propagation have been undertaken by several authors, mostly restricted to axisymmetric (or 2.5D) configurations. Recently, 3D simulations of magnetized "spine-sheath" jets have emerged as well (Mizuno et al. 2007), for purely axial magnetic field configurations at modest Lorentz factors of $\Gamma=2.5$. Combined with linear stability results for relativistic "top-hat" profiles (i.e. uniform jet bounded by a uniform sheath), these results confirm the possibility of jet stabilization by invoking radial structure. We restrict our study to axisymmetric configurations (as even our gridadaptive simulations are still fairly computationally expensive for long-term evolutions), but emphasize high Lorentz factor flow regimes in helical field topologies. Original RMHD simulations were presented by van Putten (1996), where low Lorentz factors in toroidally magnetized jets were simulated. A significant step forward was presented by Komissarov (1999), with studies of light, Lorentz factor $\Gamma=10$ jets pervaded by purely toroidal magnetic fields. By contrasting a Poyntingflux-dominated, with a kinetic-energy-dominated jet, clear morphological differences were identified and explained: extensive cocoons form in kinetic-energy dominated cases, while "nose cones" develop with high stand-off distances between Mach disk and bow shock for the Poynting-dominated jets. The latter correlate to highly magnetized, shocked plasma, self-collimated by strong magnetic pinching. These two distinctive cases have been used subsequently by various authors, also as benchmarks for the rapidly growing code parc capable of performing special relativistic MHD computations (Keppens \& Meliani 2007; Mignone et al. 2005), while a first more comprehensive study was presented by Leismann et al. (2005), also including purely poloidally magnetized jets. They confirm the purely toroidal field cases, but also demonstrate that purely poloidal field cases did not develop pronounced nose cones. The purely poloidal cases were found less susceptible to internal shock deformations, due to the stabilizing magnetic tension, hence leading to fairly smooth cocoons and stable beams. The authors speculated how the resulting brightness differences would allow inherent magnetic topologies to be distinguished. We here revisit this suggestion by making a more concentrated effort on kinetic-energy-dominated jets alone, with a more gradual change from toroidal to near poloidal fields. The helical field configurations presented indeed confirm the earlier trends, but our gridadaptive models do show rich shock-structured beams for more poloidal field regimes. The code used here is the AMRVAC code (Keppens et al. 2003; Meliani et al. 2007; van der Holst \& Keppens 2007), which has also been tested on a variety of stringent $1 \mathrm{D}$ and multi-D relativistic MHD problems (van der Holst et al. 2008). In Sect. 2, we list the governing equations, Sect. 3 provides details on the numerical strategy, while Sect. 4 discusses the main results.

\section{Magnetohydrodynamics in special relativity}

In special relativistic theory, where material particles move through four-dimensional spacetime with speeds strictly less than the speed of light $c$, the governing conservation laws express particle-number conservation and energy-momentum conservation expressable as the vanishing divergence of a stressenergy tensor. This four-tensor includes (perfect) gas, as well as electromagnetic contributions, and in general, the full dynamics governed by gas and electromagnetic field variations also needs to solve the full set of Maxwell equations. When we adopt the ideal MHD approximation, in which the electric field in the comoving frame vanishes identically, a numerically convenient set of conservation laws results when we choose a laboratory Lorentzian reference frame with four-coordinates $(c t, \boldsymbol{x})^{\mathrm{T}}$, where $\boldsymbol{x}=\left(x_{1}, x_{2}, x_{3}\right)$ are the three spatial orthogonal coordinate axes. The spacetime metric for special relativistic applications is the usual Minkowski metric $g^{\alpha \beta}=\operatorname{diag}(-1,1,1,1)$, where the Greek indices take values from $0,1,2,3$. If we indicate the proper density as experienced in the local rest frame with $\rho=m_{0} n_{0}$, where $m_{0}$ denotes the particle rest mass and $n_{0}$ the proper number density, Lorentz contraction results in a laboratory frame number

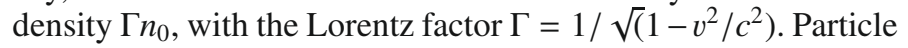
number conservation as written for the fixed reference frame is then written using the variable $D=\Gamma \rho$ as

$\frac{\partial D}{\partial t}+\nabla \cdot(D v)=0$

This includes the three-velocity $\boldsymbol{v}$, whose magnitude $v<c$. The temporal component of the divergence of the stress energy can be written in the same laboratory frame as

$\frac{\partial}{\partial t}\left(\tau+D c^{2}\right)+\nabla \cdot S=0$

The energy-flux $S$ expression is given below, and the total energy in the laboratory frame is then split off in a rest-mass contribution $D c^{2}$, and the partial energy $\tau$ made up of gas, magnetic and electric field energy densities from

$\tau=\tau_{\mathrm{g}}+\frac{B^{2}}{2 \mu_{0}}+\epsilon_{0} \frac{E^{2}}{2}$ 
In Eq. (3), $B$ and $E$ indicate the magnitude of the usual threevector magnetic $\boldsymbol{B}$ and electric $\boldsymbol{E}$ fields, respectively. When we adopt a simplifying polytropic equation of state where the specific internal energy of the gas is directly related to the proper (rest frame) density and pressure, i.e. $p /(\gamma-1) \rho$ with polytropic index $\gamma$, the gas contribution is

$\tau_{\mathrm{g}}=\rho\left(c^{2}+\frac{\gamma p}{(\gamma-1) \rho}\right) \Gamma^{2}-p-D c^{2}$.

The term between brackets represents the relativistic specific enthalpy containing a rest-mass contribution. The energy flux in Eq. (2) can also be split into gas and electromagnetic contributions as in $S=S_{\mathrm{g}}+S_{\mathrm{em}}$, where the latter are then quantified from

$$
\begin{aligned}
\boldsymbol{S}_{\mathrm{g}} & =\rho\left(c^{2}+\frac{\gamma p}{(\gamma-1) \rho}\right) \Gamma^{2} \boldsymbol{v}, \\
\boldsymbol{S}_{\mathrm{em}} & =\frac{\boldsymbol{E} \times \boldsymbol{B}}{\mu_{0}} .
\end{aligned}
$$

Obviously, $\boldsymbol{S}_{\mathrm{em}}$ is the Poynting flux. With this notation, the spatial part of the stress-energy divergence, using the ideal MHD approximation, can be written in the lab frame as

$$
\frac{\partial \boldsymbol{S}}{\partial t}+\nabla \cdot\left(\boldsymbol{S} \boldsymbol{v}+p_{\mathrm{tot}} c^{2} \boldsymbol{I}-\frac{c^{2}}{\mu_{0}} \frac{\boldsymbol{B} \boldsymbol{B}}{\Gamma^{2}}-\frac{(\boldsymbol{v} \cdot \boldsymbol{B})}{\mu_{0}} \boldsymbol{v} \boldsymbol{B}\right)=0 .
$$

The $\boldsymbol{I}$ denotes the three-by-three identity matrix, and the total pressure $p_{\text {tot }}$ is computed from

$p_{\text {tot }}=p+p_{\text {mag }}=p+\frac{1}{2 \mu_{0}}\left(\frac{\boldsymbol{B} \cdot \boldsymbol{B}}{\Gamma^{2}}+\frac{(\boldsymbol{v} \cdot \boldsymbol{B})^{2}}{c^{2}}\right)$.

The system is then closed with the homogeneous Maxwell equations

$$
\begin{aligned}
\nabla \cdot \boldsymbol{B} & =0, \\
\frac{\partial \boldsymbol{B}}{\partial t} & =\nabla \times(\boldsymbol{v} \times \boldsymbol{B}) .
\end{aligned}
$$

This equality is completely identical to the non-relativistic induction equation in ideal MHD formulations, since vanishing electric fields in the comoving frame similarly imply $\boldsymbol{E}=-\boldsymbol{v} \times \boldsymbol{B}$. This allows writing the electric energy density in Eq. (3) as $\epsilon_{0} E^{2} / 2=\epsilon_{0}\left[B^{2} v^{2}-(\boldsymbol{v} \cdot \boldsymbol{B})^{2}\right] / 2$, and the Poynting flux as $\boldsymbol{S}_{\mathrm{em}}=$ $\left[B^{2} \boldsymbol{v}-(\boldsymbol{v} \cdot \boldsymbol{B}) \boldsymbol{B}\right] / \mu_{0}$.

\section{Numerical approach}

\subsection{Algorithmic details}

For the numerical solution of the relativistic MHD equations, we actually combine Eq. (2) and the particle conservation law Eq. (1) to obtain the following conservation law

$$
\frac{\partial \tau}{\partial t}+\nabla \cdot\left(\left(\tau+p_{\text {tot }}\right) \boldsymbol{v}-\frac{(\boldsymbol{v} \cdot \boldsymbol{B})}{\mu_{0}} \boldsymbol{B}\right)=0 .
$$

It is then also necessary for the numerical approach to exploit a scaling where $c=1$, thus setting electromagnetic units where $\mu_{0}=1=\epsilon_{0}$. This scaling will be exploited from here onwards. In terms of the conserved variables $[D, S, \tau, \boldsymbol{B}]$, the usual nonrelativistic ideal MHD equations in terms of the conserved quantities $\left[\rho, \boldsymbol{m} \equiv \rho \boldsymbol{v}, e \equiv \rho v^{2} / 2+p /(\gamma-1)+B^{2} / 2, \boldsymbol{B}\right]$ are obtained directly from the limit $\Gamma \rightarrow 1$. In our conservation-law-oriented integration strategy, the induction equation (8) is written as

$\frac{\partial \boldsymbol{B}}{\partial t}+\nabla \cdot(\boldsymbol{B} \boldsymbol{v}-\boldsymbol{v} \boldsymbol{B})=\eta_{d} \nabla(\nabla \cdot \boldsymbol{B})$.

Coefficient $\eta_{d}$ is chosen to correspond to the maximal allowed diffusion coefficient, which still complies with an unmodified Courant-Friedrichs-Lewy constrained time step $\Delta t$. This acts to diffuse potential numerical monopole errors at their maximal rate, and it has been used in various non-relativistic MHD applications. We refer to Keppens et al. (2003) for a comparitive study between this and other popular source term strategies for $\nabla \cdot \boldsymbol{B}$ treatments in an AMR framework and to van der Holst \& Keppens (2007) for its use in AMR in combination with curvilinear coordinates.

When we then introduce the auxiliary variable $\xi$ from

$\xi=\Gamma^{2}\left(\rho+\frac{\gamma p}{(\gamma-1)}\right)$,

the Lorentz factor in essence depends on $\Gamma(\boldsymbol{S}, \boldsymbol{B} ; \xi)$, as one can write $\boldsymbol{v}=\left(\boldsymbol{S}+\xi^{-1}(\boldsymbol{S} \cdot \boldsymbol{B}) \boldsymbol{B}\right) /\left(\xi+B^{2}\right)$. The defining relation Eq. (3) then becomes

$\xi-\frac{\gamma-1}{\gamma} \frac{(\xi-\Gamma D)}{\Gamma^{2}}+B^{2}-\frac{1}{2}\left[\frac{B^{2}}{\Gamma^{2}}+\frac{(\boldsymbol{S} \cdot \boldsymbol{B})^{2}}{\xi^{2}}\right]-\tau-D=0$.

A root-finding algorithm (Newton-Raphson) is implemented to compute $\xi$ from this expression. This then suffices to make the needed conversions from conservative to primitive variables $[\rho, \boldsymbol{v}, p, \boldsymbol{B}]$.

The shock-capturing conservative discretization (with the monopole source term strictly speaking destroying perfect conservation only for $\boldsymbol{B}$ ) is then a TVDLF (Tóth \& Odstrčil 1996) type method, in which only the maximal physical propagation speed needs to be computed. This is done using the (slightly modified from Numerical Recipes) Laguerre method to compute both speed pairs pertaining to slow and fast magneto-acoustic waves as the roots of a quartic polynomial, see e.g. del Zanna et al. (2003). Since all 4 roots $\lambda$ must lie in the interval ] $-1,+1[$ but can become notoriously close to each other and unity, it helps to transform (Bergmans et al. 2005) to a variable $\mu=1 /(1-\lambda)$ with well-seperated roots on $] 0.5,+\infty[$. The TVDLF scheme is used with a Hancock predictor and is second-order accurate for smooth solutions. This implies limited linear constructions to obtain cell-edge from cell-center quantities, and in this process we employ the set $[\rho, \Gamma \boldsymbol{v}, p, \boldsymbol{B}]$.

\subsection{AMR strategy and numerical setup}

All jet simulations are done for a domain size $[R, Z] \in$ $[0,40] \times[0,200]$ and allow for six grid levels (including the base level), achieving an effective resolution of $3200 \times 8000$. The jet internal structure is specified in detail below, and it initially occupies only the region $\left[0, R_{\mathrm{j}}\right] \times\left[0, Z_{\mathrm{j}}\right]$. The exterior region will always represent a higher density medium in our simulations and is meant to mimic a denser "cloud", leading to jet deceleration by entrainment and dissipation, at least on a kpc scale. Our normalization always sets the jet radius to $R_{\mathrm{j}}=1.5$ and height to $Z_{\mathrm{j}}=3$. We typically compute till times beyond $t=210$; and due to the adopted scaling where the light speed $c=1$, we thus follow jet propagation for at least 70 light crossing times of the jet beam diameter $2 R_{\mathrm{j}}$. To convert from dimensionless, computed values to physical quantities, one may adopt (Harris \& Krawczynski 2006) 
a typical radius for an AGN jet of $0.05 \mathrm{pc}$, a cloud number density of $10 \mathrm{~cm}^{-3}$ and the speed of light. Our AMR scheme exploits a Richardson-type error estimator to dynamically create or remove finer level grids where needed. In addition to this estimator, we always enforce the highest grid level around this inlet corner, such that we have 120 grid cells through the jet radius $R_{\mathrm{j}}$ and the same amount of cells through the initial jet height $Z_{j}$. In the Richardson process itself, two low-order $t^{n+1}$ solutions with coarsened grid spacing $2 \Delta x$ are constructed from the known solutions at resolution $\Delta x$ at times $t^{n}$ and $t^{n-1}$, by reversing the order of the time integration and coarsening operations. When a weighted average of selected components exceeds a tolerance parameter $\epsilon_{\mathrm{tol}}=0.005$, new grids are created. In all runs, these selected components include the lab "density" $D$, partial energy density $\tau$, and magnetic field component $B_{\varphi}$, with weight ratios $2: 1: 1$

The boundary conditions for the simulations enforce the primitive variable profiles as discussed in the next section within $R<R_{\mathrm{j}}$ at the lower boundary. The remainder of this lower $Z=0$ boundary is treated as a symmetry boundary for $D, S_{R}, S_{\varphi}, \tau$, and $B_{Z}$, while we ensure the vanishing of $S_{Z}, B_{R}, B_{\varphi}$. This acts as a kind of reflecting underlying "disk" configuration from which potential backflows deflect. The lateral and top boundaries are open, while the symmetry axis $R=0$ enforces the usual (a)symmetry conditions.

\section{AGN jet computations}

\subsection{Jet inlet conditions}

In all 8 simulations discussed below, we fix the polytropic index $\gamma=5 / 3$. In fact, all models investigated in this paper represent underdense jets and typically have maximum Lorentz factor at the axis of 22. Then, we can expect from analogous 1D Riemann problems that the forward shock and reverse shock are both near Newtonian, so that it is quite adequate to use this Newtonian polytropic index value. The magnetic configuration in the lab frame is initially given by

$$
\begin{aligned}
& B_{R}=2 B_{0} \frac{R_{\mathrm{j}}}{Z_{\mathrm{j}}} \frac{\left(\frac{Z}{Z_{\mathrm{j}}}\right)^{3} \tanh \left(\frac{Z}{Z_{\mathrm{j}}}\right)^{4} \tanh \left(\frac{R}{R_{\mathrm{j}}}\right)^{2}}{\frac{R}{R_{\mathrm{j}}} \cosh \left(\frac{Z}{Z_{\mathrm{j}}}\right)^{4}}, \\
& B_{Z}=B_{\mathrm{c}}+\frac{B_{0}}{\left[\cosh \left(\frac{R}{R_{\mathrm{j}}}\right)^{2}\right]^{2} \cosh \left(\frac{Z}{Z_{\mathrm{j}}}\right)^{4}} \text {, } \\
& B_{\varphi}=\left\{\begin{array}{lr}
B_{1} \tanh \left(\frac{R}{a}\right) & \text { in } \quad\left[0, R_{\mathrm{j}}\right] \times\left[0, Z_{\mathrm{j}}\right], \\
0 & \text { elsewhere. }
\end{array}\right.
\end{aligned}
$$

This solenoidal field is fully helical internal to the jet whenever $B_{1} \neq 0$, while it is purely poloidal elsewhere. The parameter " $a$ " for the azimuthal field variation is held fixed at $a=5$; and in all but our purely toroidal field simulation (where the exterior is unmagnetized), the magnetic field strength of the cloud $B_{\mathrm{c}}=0.01$, corresponding to a weak uniform background magnetic field. We explore the relative importance of the jet internal toroidal versus poloidal field components by varying the parameters $B_{0}$ and $B_{1}$. The proper densities of the jet and cloud are typically fixed at $\rho_{\mathrm{j}}=100$ and $\rho_{\mathrm{c}}=1000$, except for one run where the cloud density $\rho_{\mathrm{c}}$ is decreased twofold. Hence, we restrict the discussion to the propagation of relativistic underdense jets, since they yield deceleration of the jet and formation of the typically complex cocoons. Moreover, we only explore relativistic jets dominated by
Table 1. Model input parameters, as appearing in the profiles quantifying the radial variation of primitive variables.

\begin{tabular}{lcccc}
\hline \hline Model & $\rho_{\mathrm{j}} / \rho_{\mathrm{c}}$ & $B_{0}, B_{\mathrm{c}}, B_{1}$ & $\alpha$ & $p_{\mathrm{j}}$ \\
\hline NR & 0.1 & $1,0.01,1$ & 4.99 & 1 \\
MR & 0.1 & $1,0.01,1$ & 9.9 & 1 \\
Ref1 & 0.1 & $1,0.01,1$ & 9.99 & 1 \\
\hline Pol & 0.1 & $1,0.01,0.01$ & 999.9 & 1 \\
Tor & 0.1 & $0,0,1$ & 9.99 & 1 \\
Ref2 & 0.1 & $2,0.01,1$ & 9.99 & 1 \\
\hline Ref3 & 0.2 & $1,0.01,1$ & 9.99 & 1 \\
Ref4 & 0.1 & $1,0.01,1$ & 9.99 & 2 \\
\hline
\end{tabular}

kinetic energy, which are then characterized by a strong shock (Appl \& Camenzind 1988), which in turn are efficient in particle acceleration (Begelman \& Kirk 1990). The initial pressure distribution is computed from

$p=p_{\mathrm{j}}+\frac{1}{2} B_{0}^{2}-\frac{1}{2}\left(B_{\varphi}^{2}(R, Z)+B_{Z}^{2}(R, Z)\right)$,

where the reference value for the parameter $p_{\mathrm{j}}=1$ (representing a cold jet). Finally, the initial flow field $v$ is quantified by the prescription

$$
\begin{aligned}
& v_{Z}=\alpha \frac{B_{\varphi}}{\sqrt{\rho}(R / a)}, \\
& v_{\varphi}=\frac{B_{\varphi}}{\sqrt{\rho}} \\
& v_{R}=0 .
\end{aligned}
$$

It vanishes outside the jet region $\left[0, R_{\mathrm{j}}\right] \times\left[0, Z_{\mathrm{j}}\right]$ and includes jet rotation whenever $B_{1} \neq 0$. Note that the requirements $p>0$ and $v<1$ restrict the possible choices for the parameters $p_{\mathrm{j}}$ and $\alpha$, once values for $B_{0}, B_{1}\left(B_{\mathrm{c}}\right)$ and $\rho_{\mathrm{j}}$ are adopted. This prescription for the primitive variables $\rho, \boldsymbol{v}, p$, and $\boldsymbol{B}$ is inspired by previous non-relativistic ideal MHD models as exploited in Casse \& Marcowith (2005) and is such that it then ensures the radial force balance along the lower boundary $Z=0$. In what follows, we quantify how these parameters translate into various dynamically important dimensionless parameters like the Lorentz factor, Mach number, Alfvén Mach number, and magnetization parameter, as averaged over the jet radius. In our implementation, we find it convenient to prescribe these jet profiles at the inlet boundary $Z=0$ directly from the above primitive variable expressions within those bottom ghost cells with cell centers $Z<0$ and $R<R_{\mathrm{j}}$.

In Table 1, we list the input model parameters for the eight cases studied. The first three runs only differ in the value of $\alpha$, and they mimic the effect of going from nearly non-relativistic speeds (NR) to mildly relativistic (MR) to fully relativistic flows in our reference case (Ref1). The next three models (Pol, Tor, Ref2) explore variations in magnetic field topology (from almost purely poloidal to fully toroidal). The final two cases look at modest changes (factor 2) in external density (Ref3) or overall pressure (Ref4). Note that, for all the cases considered, the entire jet rotation profile enforced at the inlet $Z=0$ is within the light cylinder: i.e., within the jet radius $R<R_{\mathrm{j}}$, we have $\left|v_{\varphi}-v_{Z} B_{\varphi} / B_{Z}\right|$ increasing from zero to 0.63 for most of the models. In Table 2 , we list the corresponding dimensionless parameters that characterize the internal jet properties. We list the Lorentz factor $\Gamma$, the Mach number $M=v_{Z} / c_{\mathrm{s}}$ with the sound speed given by $c_{\mathrm{s}}=\sqrt{\gamma(\gamma-1) p /((\gamma-1) \rho+\gamma p)}$, and the relativistic proper Mach number $\mathcal{M}=M \Gamma / \Gamma_{\mathrm{s}}$, where $\Gamma_{\mathrm{s}}=1 / \sqrt{\left(1-c_{\mathrm{s}}^{2}\right)}$ is the 
Table 2. Model parameters, in terms of beam-averaged inlet values for various dimensionless ratios.

\begin{tabular}{lccccc}
\hline \hline Model & $\bar{\Gamma}$ & $\overline{\beta_{\mathrm{r}}}$ & $\bar{\sigma}$ & $\bar{M}, \overline{\mathcal{M}}$ & $\overline{M_{\mathrm{a}}}$ \\
\hline NR & 1.15 & 0.3015 & 0.0067 & $3.6,4.1$ & 6.6 \\
MR & 4.79 & 0.2902 & 0.0064 & $7.1,34.2$ & 13.6 \\
Ref1 & 6.92 & 0.2899 & 0.0064 & $7.2,50.3$ & 13.7 \\
\hline Pol & 7.78 & 0.2833 & 0.0063 & $7.2,56.9$ & 13.9 \\
Tor & 6.92 & 0.0009 & 0.00002 & $7.8,53.3$ & 664.5 \\
Ref2 & 6.92 & 0.9147 & 0.0250 & $6.2,44.7$ & 7.1 \\
\hline Ref3 & 6.92 & 0.2899 & 0.0064 & $7.2,50.3$ & 13.7 \\
Ref4 & 6.92 & 0.1514 & 0.0064 & $5.3,36.5$ & 13.9 \\
\hline
\end{tabular}

Lorentz factor computed from the sound speed. We then give the Alfvén Mach number $M_{\mathrm{a}}=v_{Z} / c_{\mathrm{a}}$ where the Alfvén speed is found from (Lichnerowicz 1967)

$c_{\mathrm{a}}=\sqrt{\frac{\sigma}{\sigma+1+\frac{\gamma p}{(\gamma-1) \rho}}}$.

This equation contains the ratio of magnetic to rest-mass energy density

$\sigma=\frac{2 p_{\mathrm{mag}}}{\rho}$,

which together with the reciprocal plasma- $\beta$ parameter,

$\beta_{\mathrm{r}}=\frac{p_{\mathrm{mag}}}{p}$,

is known to be an important parameter characterizing the morphological appearance of jets with purely toroidal magnetic field configurations, where we follow the definitions from Leismann et al. (2005). For such jets, the pioneering work by Komissarov (1999) has shown that Poynting-flux-dominated (high $\sigma$ ), strongly magnetized (high $\beta_{\mathrm{r}}$ ) jets may develop a sharp "nose cone", where highly magnetized plasma accumulates beyond the terminal shock of the jet beam. In contrast, strong backflows, therefore cocoon-dominated jets, emerged when the kinetic energy flux is dominant (low $\sigma$ ). In the Leismann et al. (2005) parameter study that extends these results to also purely poloidal field configurations, no "nose cones" were found for poloidal topologies.

Due to the 2.5D nature of the equilibrium, all these parameters in fact vary from mildly to strongly across the beam radius. In Table 2, we computed beam-averaged values

$\bar{f}=\frac{1}{\pi R_{\mathrm{j}}^{2}} \int_{0}^{R_{\mathrm{j}}} 2 \pi R f \mathrm{~d} R$.

From the values in this table, the sequence of models NR, MR, Ref1 with increasing $\alpha$ clearly has similar inlet conditions (low $\sigma$, equipartition like fields with $\beta_{\mathrm{r}} \approx 0.3$ ) and explores the trend to $\bar{\Gamma} \approx 7$ flow regimes. In Fig. 1 , we plot the actual variation of the Lorentz factor $\Gamma$ across the inlet for the Ref1 model, and it is seen that this model reaches axial flows with $\Gamma$ up to 22 . The figure also shows the inverse pitch $\mu$ for the same model. The inverse pitch $\mu$ is a quantitative measure of the twist in the jet magnetic field and is defined from

$\mu=\frac{R_{\mathrm{j}} B_{\varphi}}{R B_{Z}}$.

This profile is important for a stability analysis of helically magnetized jet flows; and as we have axial values of this inverse
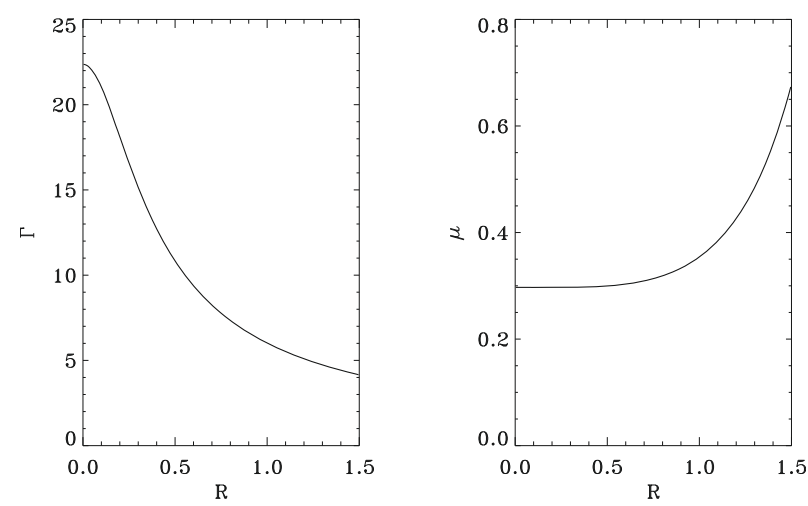

Fig. 1. Jet inlet profiles for the reference model Ref1. Left: radial variation of the Lorentz factor $\Gamma$. Right: variation in the inverse pitch $\mu$.

pitch of order 0.3 and as the current is distributed over the entire jet section (Appl et al. 1999), we may expect that nonaxisymmetric current-driven instabilities are less likely to play a role in the dynamics. However, a quantitative answer to stability with respect to non-axisymmetric perturbations (both of KelvinHelmholtz as well as current-driven type) is presently lacking for radially structured jets such as those introduced here. While simplified "top-hat" profiles (i.e. layers of uniform, axially magnetized flows) can still be treated analytically (Hardee 2007), a numerical analysis of the linearized relativistic MHD equations is needed to predict 3D effects which we artificially suppress by axisymmetry assumption. Naturally, the sequence of models Pol, Tor, Ref2 with varying field structure will be characterized by very different stability properties against non-axisymmetric modes.

As a means of anticipating the $2.5 \mathrm{D}$ results and verifying whether the employed resolution suffices to capture the flow details, we first show the result of solving two Riemann problems that relate to the initial conditions in the reference case (Ref1). We solve them numerically using essentially identical settings for grid refinement and resolution. The on-axis conditions, where Lorentz factor $\Gamma \approx 22$ conditions prevail, are as in the $1 \mathrm{D}$ problem where for constant $B_{x}=1$, left state L1 is adjacent to the right state $\mathrm{R} 1$ as given by

\begin{tabular}{cccccc}
\hline & $\rho$ & $p$ & $v_{x}$ & $v_{y}$ & $B_{y}$ \\
\hline$L 1:$ & 100 & 1 & 0.999 & 0 & 0 \\
$R 1:$ & 1000 & 1.5 & 0 & 0 & 0 \\
\hline$L 2:$ & 100 & 1 & 0.992 & 0.015 & 0.15 \\
$R 2:$ & 1000 & 1.5 & 0 & 0 & 0 \\
\hline
\end{tabular}

States L2 and R2 give a Riemann problem that relates to the conditions at radius $R \approx 0.75$, midway through the jet. It must be noted that this relation is somewhat ad hoc, since the $2 \mathrm{D}$ case has significant variation of $B_{Z}(Z)$ and $p(Z)$ at the head of the jet, which cannot be incorporated in a 1D Riemann problem. Nevertheless, Figs. 2, 3 show the result at $t \approx 200$ for these Riemann problems. In all cases, we capture both fast, forward and reverse shocks very accurately, despite the fairly extreme parameters. The contact discontinuity is smeared over many cells, but adequately resolved thanks to the AMR. More problematic is the separation between contact discontinuity and the varying tangential field and velocity components in the Alfvén signals in the second Riemann problem. Still, their amplitude and variation are appropriately represented (and the entropy is constant through these discontinuities). These 1D tests qualify the shockrelated pressure variations to be several orders of magnitude and indicate that we will be able to follow jet dynamics on a similar timescale within the computational domain. 

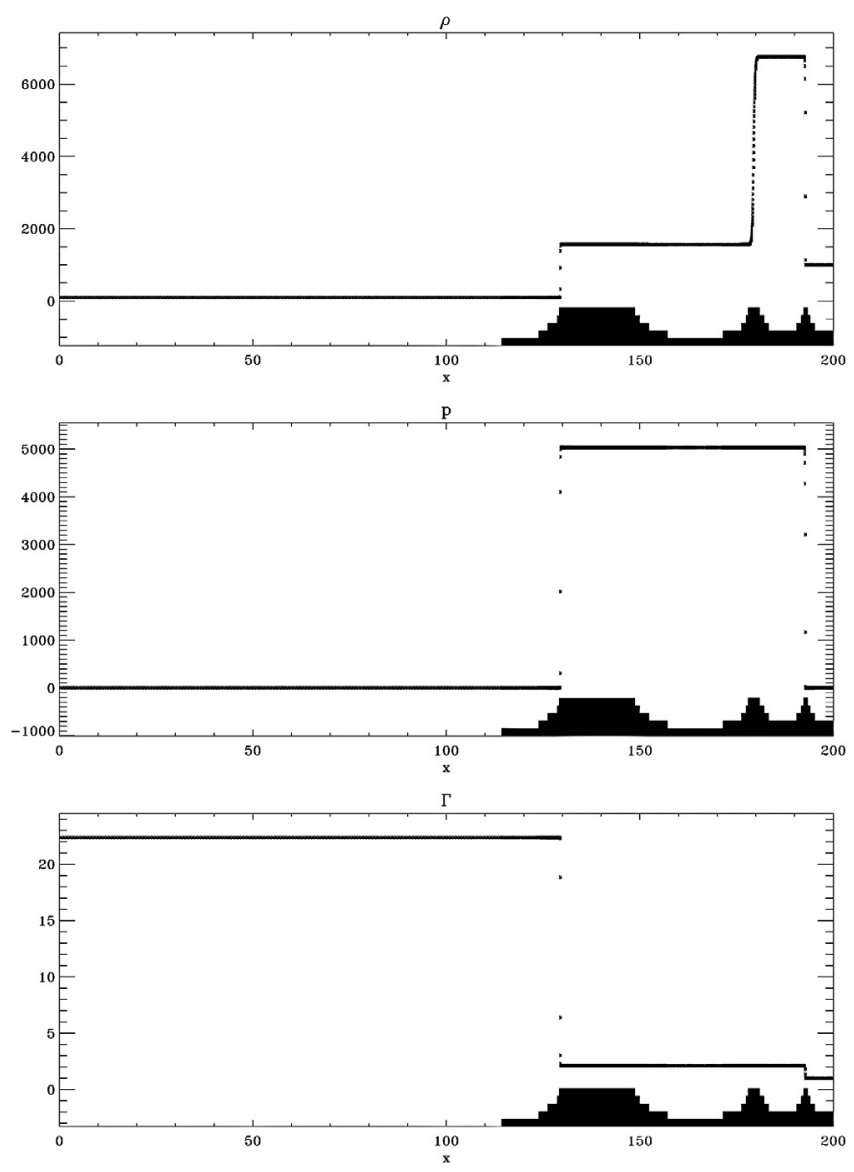

Fig. 2. A 1D Riemann problem indicative of the on-axis conditions of the reference jet. Shown are the proper density, pressure, and Lorentz factor, at time $t \approx 200$.

\subsection{Jet morphologies: from non-relativistic to relativistic}

To qualify the overall jet morphologies, we used the sequence of models characterized by increasing velocity (NR, MR, Ref1). In the near non-relativistic model NR, the jet beam terminates in a Mach disk, across which a fair part of the directed kinetic energy is transferred to internal energy. This gives rise to a prominent hot spot of high pressure material, typically extending up to the contact discontinuity (or working surface) between shocked jet material and shocked cloud material. The magnetic field is compressed by the reverse shock (Mach disk), enhancing the collimation efficiency that limits the sideways expansion of the shocked beam matter (in between Mach disk and working surface). The shocked cloud material is bounded by an overarching bow shock. In this NR model, the inertia ratio between the jet and the external medium is low $\eta \sim \bar{\Gamma}_{\mathrm{b}}^{2} \rho_{\mathrm{b}} / \rho_{\mathrm{c}}=0.132$, so that we expect turbulent behavior near the working surface, which will lead to fairly strong disturbances to the jet. The compression of the shocked external medium is moderate, and the sideways spreading of shocked beam material is reflected by the external medium leading to a bow shock and backflow. In animated views of the jet evolution, one can witness the formation of vortical patterns at the contact interface in front of the Mach disk, which get pushed away laterally and form complex backflow patterns surrounding the jet beam proper. In doing so, recurrent cross-shocks are driven into the beam in the region behind the terminal shock. These lead to interacting diagonal cross-shock patterns internal to the beam, and they continually restructure the shape and lateral extent of the Mach disk at the end of the beam. A plot at
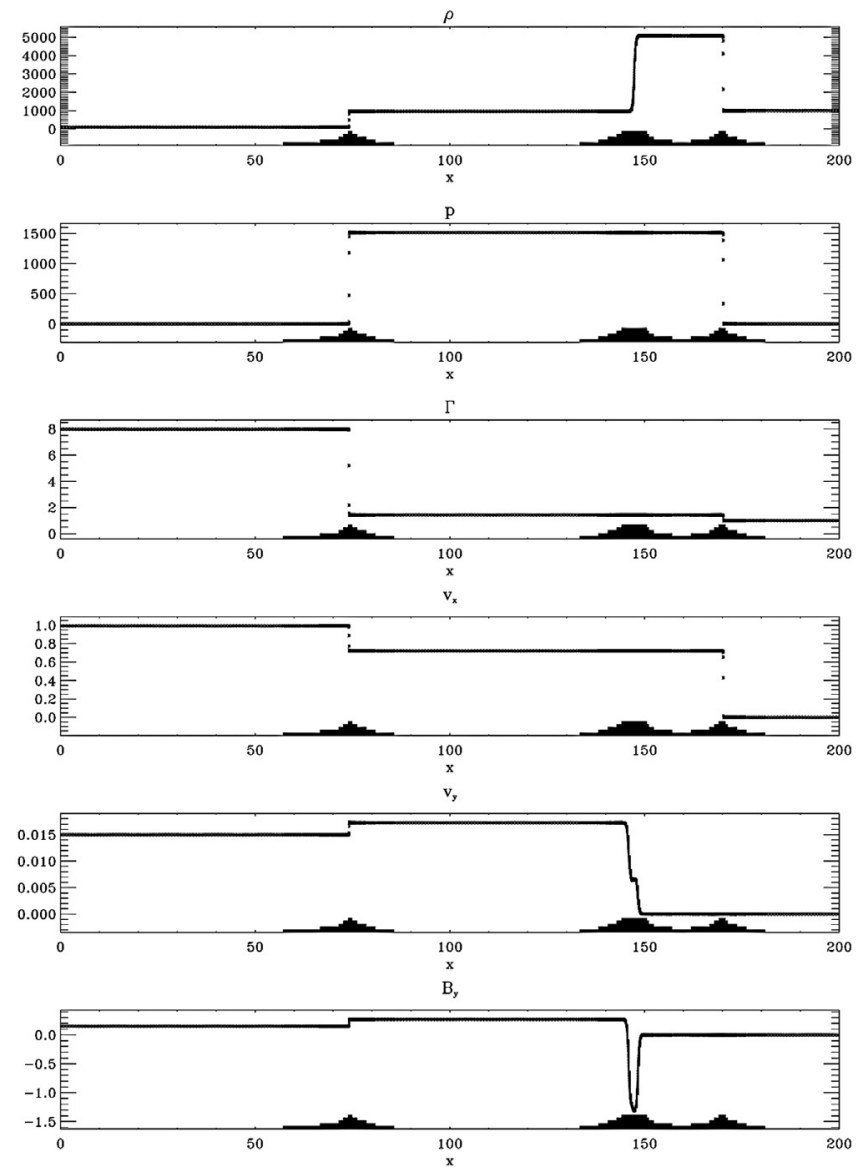

Fig. 3. A $1 D$ Riemann problem indicative of $R \approx 0.75$ (midway) conditions of the reference jet. Shown are the density, pressure, Lorentz factor, and $v_{x}, v_{y}$, and $B_{y}$ at time $t \approx 200$.

time $t \simeq 555$ of the pressure distribution (showing the high pressure hot spot), the reciprocal plasma parameter $\beta_{\mathrm{r}}$, and the proper density (in logarithmic scale) are all shown in Fig. 4.

The magnetic field configuration for model NR at the same time $(t=555)$ is shown in the top panel of Fig. 5. In the beam, slight helicity changes are associated with each diagonal cross-schock pattern, with increased twist regions across a converging cross shock, up to the diverging fronts. In the cocoon region formed by the backflowing material, rather strong azimuthal field components prevail, and the helically magnetized jet beam is surrounded by predominantly toroidal field regions. This can be seen in Fig. 5 where the right panel gives a view of selected field lines, combined with a translucent surface containing a Schlieren plot of the rest frame density $\rho$. In the left panel, the velocity field shows the vortical motions associated with the backflows in the cocoon. The right half of this panel maps the distribution of the absolute value of the inverse pitch $|\mu|$ from Eq. (19). Regions where $|\mu|$ exceeds unity are colored in black, and they give an indication of the most strongly wound field regions. It is seen how the $\mu$ variation across the inlet is fairly preserved throughout the jet beam up to the Mach disk, with modest variations associated with the diagonal cross shocks, as mentioned above. Beyond the Mach disk and in the cocoon, a predominant toroidal field prevails, Hence, the reverse shock (Mach disk) compresses the toroidal magnetic field. There is also clear evidence of a more poloidal, straight field layer in between the jet beam proper and the backflow regions. These backflows push the poloidal magnetic field towards the axis, and 

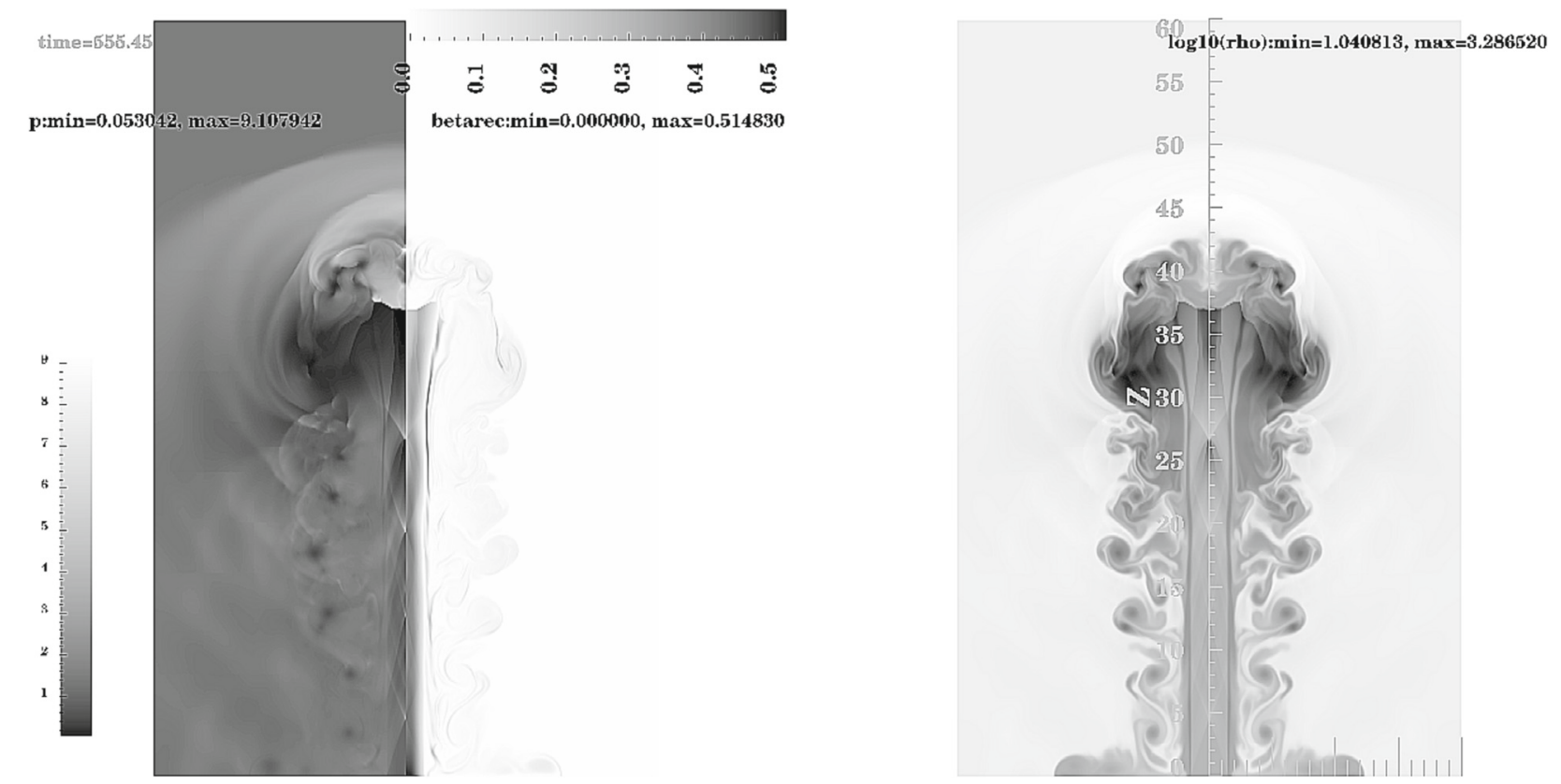

Fig. 4. For the low speed $\bar{\Gamma} \simeq 1.15$ jet (NR), we show the pressure and reciprocal plasma beta $\beta_{\mathrm{r}}($ left) and proper density (right) distribution at time $t \simeq 555$, corresponding to 185 light crossing times of the jet diameter.

the resulting layer of strong poloidal magnetic field can be expected to increase the lateral stability of the jet beam.

At all times in the evolution, only the jet beam, cocoon regions, and compressed region between beam and backflow contain any significant magnetic pressure. This is shown in Fig. 6, where we contrast the three jet models NR, MR, and Ref1 near the end of the simulated time intervals on the entire computational domain. Note that the non-relativistic model is shown at $t \simeq 555$, while the faster jets MR and Ref1 are plotted at near identical, earlier times $t \simeq 220$. The bottom half of each panel quantifies the magnetic pressure distribution $p_{\text {mag }}$ (on a logarithmic scale), and dynamically important values are found only up to the (turbulently deformed) contact interface between shocked beam plus backflow material with shocked cloud matter. This figure also shows that the faster jet models have a clearly more elongated appearance, consistent with their higher inertia $\propto \Gamma^{2}(\rho+\gamma p /(\gamma-1))$. The same figure also demonstrates that all our computations completely resolve the full bow-shock pattern because they do not (yet) cross the lateral boundary. In figures throughout this document, we show appropriately scaled, zoomed-in regions of the domain, to better illustrate flow details. In Fig. 5, the lower panel shows the magnetic field and flow information for the reference model Ref1, in the same manner as the top panel for the non-relativistic case NR. Note that times, vertical extent, and aspect ratio are quite different between the two cases, as evident from comparing them with Fig. 6. In the fast jet Ref1 (and also in the MR jet, which is not shown in Fig. 6), we again see that the magnetic field in the jet beam roughly preserves the inlet helicity profile up to the Mach disk. The beam is bordered by a near vertical field "sheet", and the vortical patterns marked by lower density structures contain the most thightly wound fields. Unlike the non-relativistic model, though, the backflowing vortices do not really form a very extended cocoon surrounding the jet beam, but appear as more isolated, narrow protrusions into the jet cavity bounded by the bow shock. The locations of the dominant toroidal field regions are therefore also fairly localized. Thin strands of high field twist thus coincide with the lowest proper density spots, which mark the centers of the vortical patterns. As will be discussed later on, the rotational flow patterns in these vortices are locally supersonic.

In the preceding discussion, we contrasted flow morphology and dynamics for mildly relativistic to strongly relativistic cases (NR to Ref1), all obtained in axisymmetric computations. All impulsively injected jet studies (where the jet gradually enters the simulation domain) tend to evolve from a $1 \mathrm{D}$ to a $2 \mathrm{D}$ phase once eddies start to dominate the cocoon dynamics and internal jet beam shocks develop fully. For the models discussed here, the first internal cross shock has already formed clearly at time $t \approx 15$. For the Ref1 case, multiple internal cross shocks, and complex (ring-like) vortex sheddings are prominent beyond times $t \approx 60$. A transition to a $3 \mathrm{D}$ phase where jet disruption and/or significant non-axisymmetric deformation occurs is precluded in our setup. According to the discussion on 2D versus 3D effects in Carvalho \& O'Dea (2002), jet disruption for classical HD jets may occur beyond distances given by $5 R_{\mathrm{j}} M_{\mathrm{j}}$, where $M_{\mathrm{j}}$ indicates the jet Mach number. If we take the jet-averaged Mach number $\bar{M}$ from Table 2, disruption to 3D can occur beyond time $t \approx 54$ and is likely to affect any further propagation. Using the more appropriate relativistic value $\bar{M} \approx \Gamma \bar{M}$, this disruption length still lies beyond the computed time interval. Earlier 3D non-relativistic hydrodynamic (e.g. Bodo et al. 1998), and magnetohydrodynamic (e.g. Baty \& Keppens 2002; O'Neill et al. 2005) studies have been able to qualify some of the consequences of the axisymmetry restriction. Bodo et al. (1998) have identified how small-scale structure emerges fast in full3D hydro simulations of periodic jet segments, and particularly light jets (just as those considered here) are very prone to nonaxisymmetric mode development. Dynamically important magnetic fields can help stabilize the jet flow and mitigate the energy cascade to smaller scales. Classical 3D MHD simulations of periodic jet segments by Baty \& Keppens (2002) identify how helically magnetized jets can maintain jet coherency despite additional global non-axisymmetric instabilities. O'Neill et al. (2005) have investigated the propagation characteristics of helically magnetized light jets in $3 \mathrm{D}$, following their propagation for 

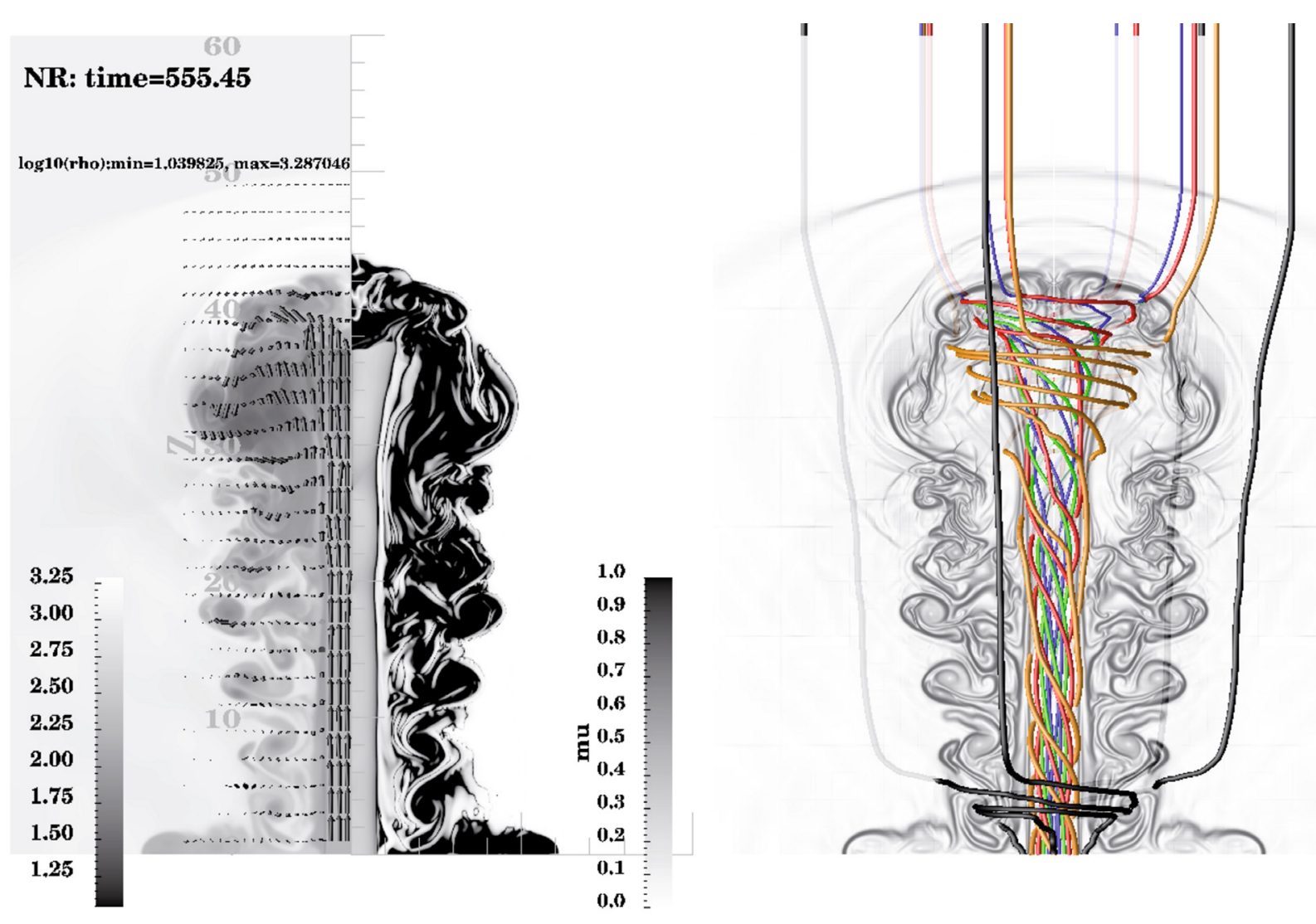

\section{Ref1: time=218.74}

$\log 10($ rho $): \min =0.693825, \operatorname{mux}=3.797852$
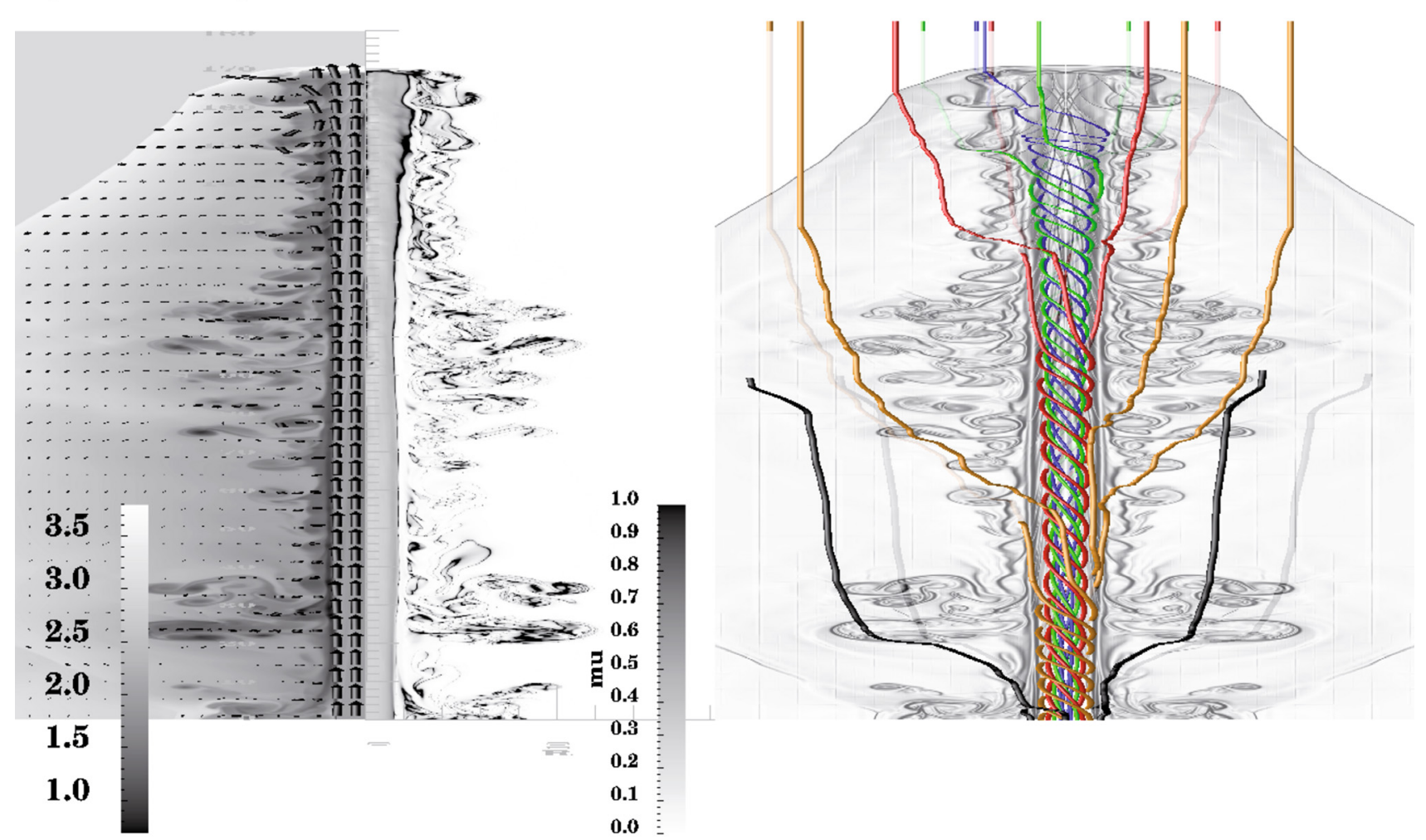

Fig. 5. For the NR jet from Fig. 4 at time $t \simeq 555$ on domain $20 \times 60$, the top panels show the flow topology on top of the density plot (leftmost panel), along with a quantification of the inverse pitch throughout the jet. Values above $\mu=1$ are colored black. In the right panel, a translucent Schlieren plot of the density is combined with field lines. The latter are colored according to their radial starting position (progressively more outwards: blue $R=0.3$, green $R=0.6$, red $R=0.9$, yellow $R=1.3$, black $R=1.8$ ). The bottom panel gives the same info for the reference model Ref1, at a different time $t \simeq 220$, using a very different aspect ratio of $20 \times 160$. 


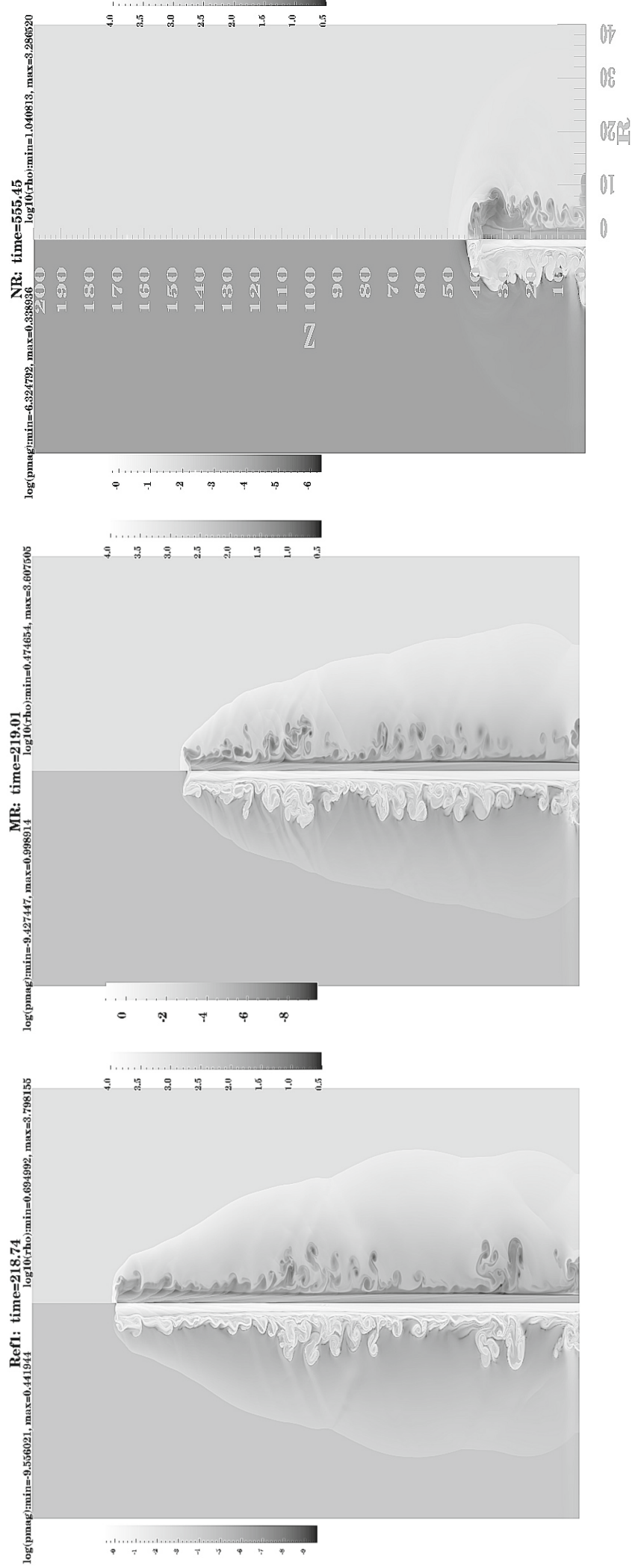

Fig. 6. The (logarithm of) magnetic pressure $p_{\text {mag }}$ (bottom) and proper density $\rho$ (top) for models NR (at $t \simeq 555$ ), and MR and Ref1 (at $t \simeq 220$ ). The aspect ratio is identical in all frames, namely $40 \times 200$.

lengths exceeding 100 jet radii. The presence of an intricately structured 3D "shock-web complex" at the frontal part of the jet was clearly seen in renderings of the compression rate, most prominent in the high Mach-number jet propagating in uniform surroundings. In view of these results, it can be expected that full 3D relativistic simulations for the sequence NR to Ref1 will show profound differences from our axisymmetric computations in the later stages of the simulation. 

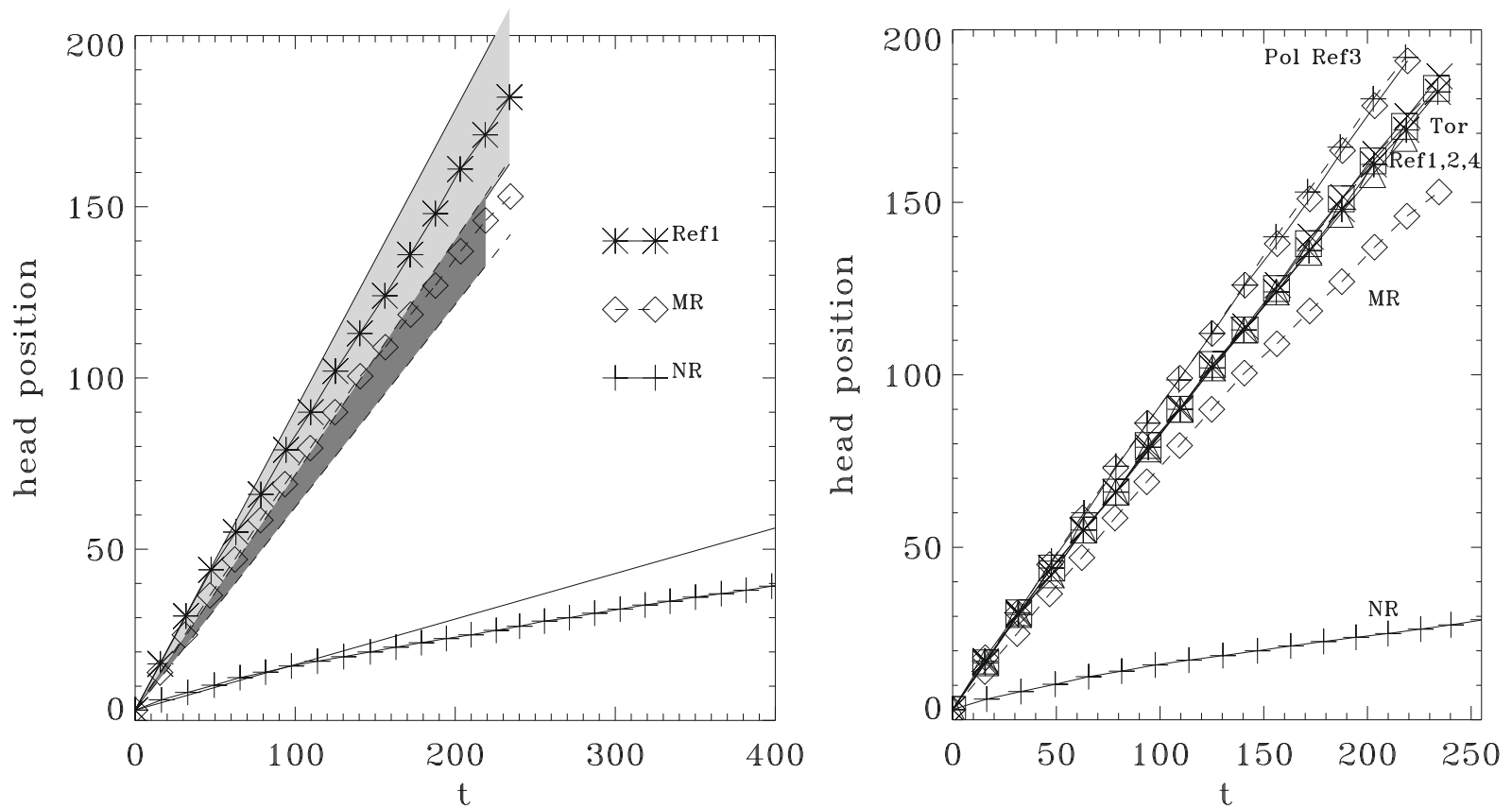

Fig. 7. The temporal evolution of the jet head position, for the sequence from non-relativistic, to the reference case (left panel), and a comparison with an approximate formula for kinetic energy dominated jets. To the right, all simulated models are shown in a similar fashion, essentially demonstrating similar propagation speeds.

\subsection{Propagation speeds}

A straightforward qualification of the jet dynamics is obtained from their propagation speeds. It was shown in relativistic hydrodynamics (Martí et al. 1997) and later used for relativistic MHD jets (Leismann et al. 2005), so that an estimate for the head advance speed could be made as

$v_{\text {head }}=\frac{\sqrt{\xi_{\mathrm{b}} / \xi_{\mathrm{a}}}}{1+\sqrt{\xi_{\mathrm{b}} / \xi_{\mathrm{a}}}} v_{Z}$

in which the jet beam internal versus (static $\Gamma_{\mathrm{a}}=1$ ) ambient medium enthalpies $\xi / \Gamma^{2}$ appear. This estimate assumes pressurematched conditions and essentially uses momentum balance in the shock frame. In our magnetized jets, all taken in the kineticenergy dominated regime $(\bar{\sigma} \ll 1$ see Table 2$)$, we can expect a reasonable agreement with this formula. Due to the internal beam equilibrium profiles, we can use this expression to get "lower" and "upper" bounds for the expected jet propagation. Using averaged beam-profile values in Eq. (21), a reasonable lower bound is obtained, while a kind of higher bound is found when specifying to beam axial values. In Fig. 7, the left panel shows these estimated speed ranges and compares them to the simulation results for the sequence of low- to high-speed jets (NR, MR, and Ref1). For the slowest jet model, both estimates essentially coincide, and this jet shows good agreement with the predicted speed up to times $t \approx 100$, after which the jet starts to propagate more slowly. For the faster MR to Ref1 models, there is also a trend toward gradual deceleration, where the upper estimate prevails in the first phase of the computation, but where we typically find actual propagation values in between the estimated bounds in the later stages. The right panel of Fig. 7 compares the computed propagation velocities for all 8 jets. The fastest jets in this limited sample are the nearly purely poloidal case, in accord with its higher beam Lorentz factor (average, as well as maximal, this time even going up to 70). In fact, this jet with (nearly) purely poloidal magnetic field behaves as a hydrodynamic jet (Majorama \& Anile 1987) in first approximation. Also the Ref3 case, which has the reference jet structure penetrating a twice lighter external medium, is fast, as it interacts weakly with the external medium. The propagation characteristics of the four other models turn out to be very similar, and this again agrees with their estimated values from Eq. (21).

Nonetheless, prominent 2D effects arise in our simulations, as e.g. seen in the shape of the bow shock in Fig. 6. This is influenced by the formation and interaction of the beam's internal cross shocks, as well as by the complex evolution of the final Mach disk. The first cross shock for the NR case forms near $Z \approx 4$ and remains approximately at this location for times up to $t \approx 300$. For the MR case, the first cross shock initially forms at about $Z \approx 12$, gradually moving to greater distances. The reference Ref 1 case initially forms a strong cross-shock at about $Z \approx 25$, but its location and strength varies with time: at $t \approx 156$, a fairly strong shock is visible at about $Z \approx 64$, but eventually a weaker shock remnant can be seen in Fig. 8 at about $Z=90$ (followed by a much stronger shock at $Z \approx 117$ ). Despite these and other highly time-varying effects at the jet head, we find that the high Mach number jets considered here thus agree well with $1 \mathrm{D}$ propagation estimates, consistent with findings for the non-relativistic, axisymmetric hydro jets presented in Carvalho \& O'Dea (2002).

In Fig. 8, we illustrate several properties of the flow field for the Ref1 reference model. Using the same overall view as displayed in Fig. 6, the bottom half of the top panel indicates where regions of locally supersonic poloidal velocities are encountered. All regions where $\sqrt{v_{R}^{2}+v_{Z}^{2}} / c_{\mathrm{S}}$ exceed unity are colored black, and the remaining greyscale is used for the subsonic range. The entire jet beam, together with the uppermost part of the bounding bow-shock is locally supersonic. The bow-shock speed varies significantly along its entire boundary. As its speed is mostly transversal, we indeed confirm that its lateral supersonic expansion varies strongly behind the jet head, as has already been found from axisymmetric hydro studies (Carvalho \& O'Dea 2002). The upper half of this panel in Fig. 8 shows the 

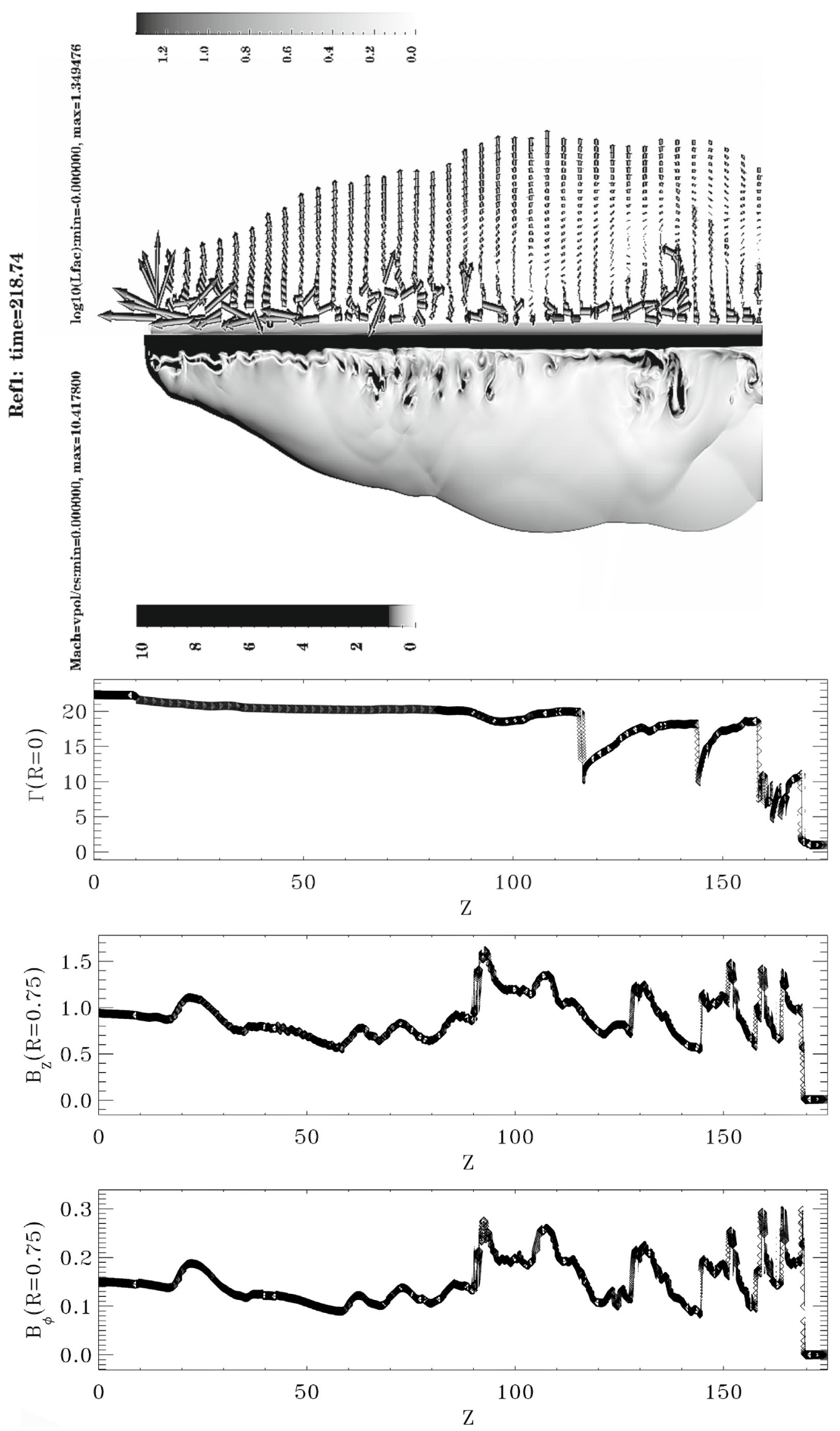

Fig. 8. For the reference Ref1 case, we visualize the flow pattern in the cocoon, along with a local Mach number quantification (bottom half of top panel). Lower three panels: we show a cut along the symmetry axis of the Lorentz factor at the same time $t \simeq 220$, a cut of $B_{R}(Z)$ and $B_{\varphi}(Z)$ at the same time for $R=0.75$. 
$\mathrm{B}_{\mathrm{k}}$
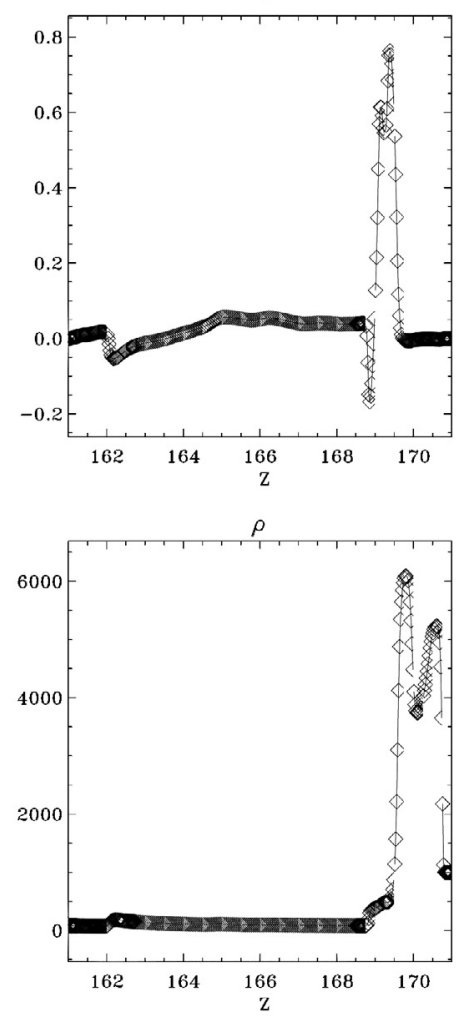

$\mathrm{S}$

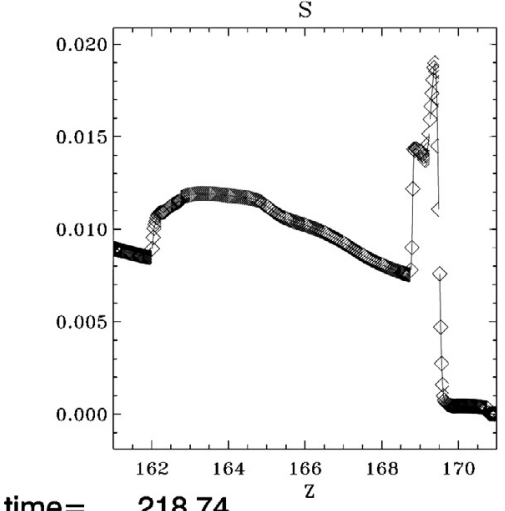

$\mathrm{B}_{\mathrm{z}}$

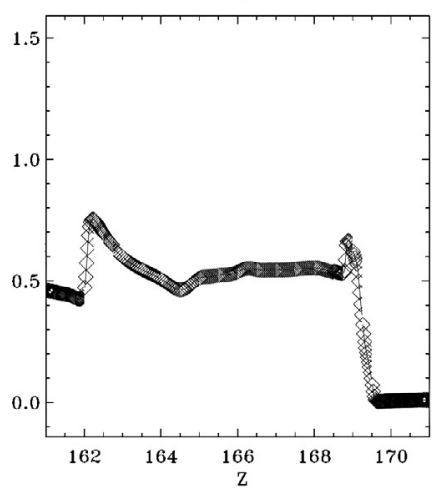

$\Gamma$

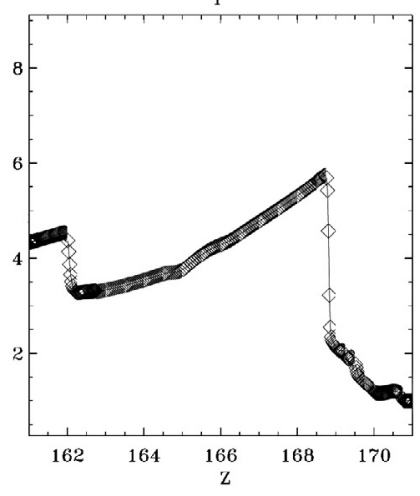

$\mathrm{v}_{\mathrm{z}}$

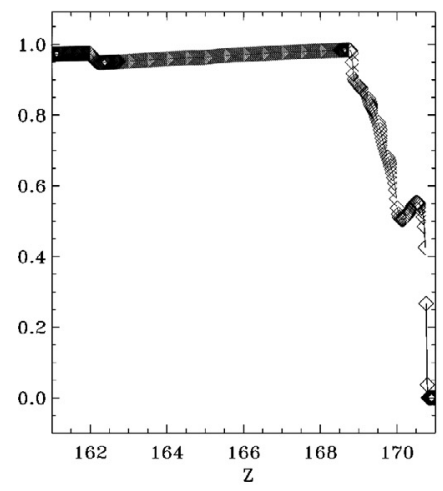

$\mathrm{B}_{\phi}$
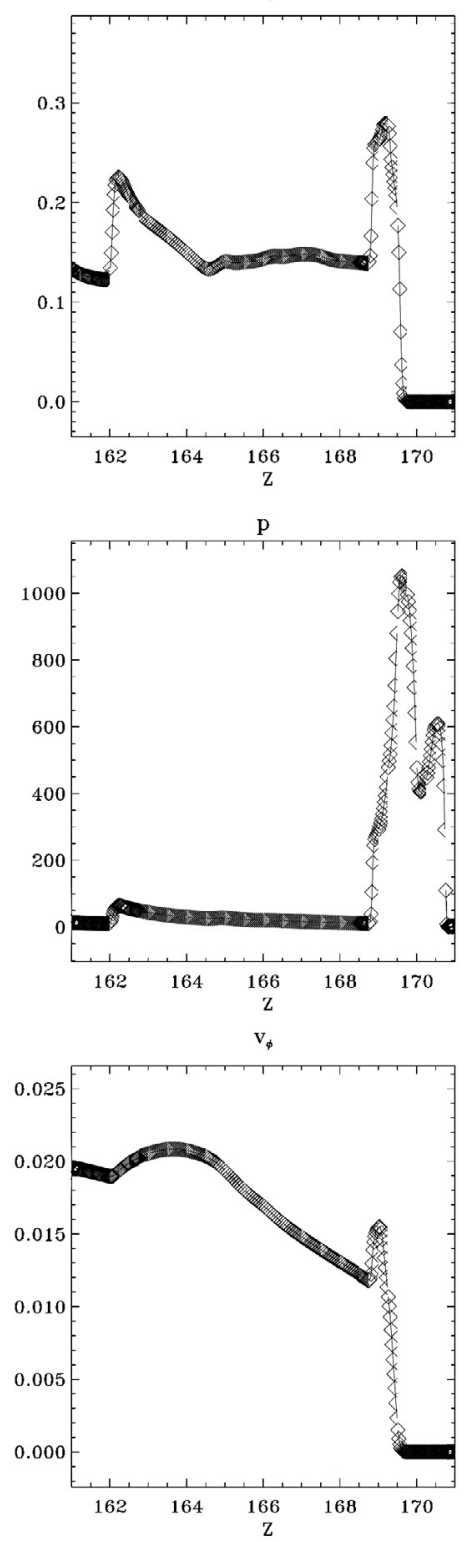

Fig. 9. For the reference jet Ref1, we show cuts through the jet head, taken at fixed radius $R=1$ and for $Z \in[161,171]$, and at the time $t \approx 220$. From left to right and top to bottom, we show field components $B_{R}, B_{Z}, B_{\varphi}$, proper density $\rho$, Lorentz factor $\Gamma$, pressure $p$, along with entropy $S$, and velocity components $v_{Z}$ and $v_{\varphi}$.

(logarithm of) the Lorentz factor in greyscale, and only the jet beam up to the Mach disk is seen to travel at a significant fraction of the speed of light (up to on-axis values $\Gamma \approx 22$, with some variation across the internal cross shocks). The velocity vectors are only drawn throughout the surrounding cavity, and they show the complex vortical patterns, the overal expansion of the cavity, and clear evidence of localized regions with strong velocity shear. The last aid in the formation of the turbulent structures by Kelvin-Helmholtz instabilities. The lower panels in Fig. 8 quantify the on-axis variation of the Lorentz factor at this time and the magnetic field variation midway the jet beam (at radius $R=0.75$ ). This shows that the beam's internal cross shocks act to convert some of the directed kinetic energy; and due to slight variations of the cross-section of the beam, matter again accelerates up to the next cross-shock. The magnetic field plays an important role in this process, because the field strongly pinches the flow downstream of the shock. Note how, in this reference case, the Lorentz factor $\Gamma \simeq 20$ flow occurs up to a distance of
$Z \simeq 158$, while at the final Mach disk, the Lorentz factor is still above 10 . Even longer simulations would be required to quantify the deceleration process better.

\section{Jet-head structure and varying magnetic topology}

If we turn to the jet-head structure in particular, we can quantify the compression occurring at the Mach disk and compare the field structure with what is obtained in the 1D Riemann problem shown in Fig. 3. Figure 9 shows the axial variation at a radial distance $R=1$, zoomed in on the jet-head structure (note the limited $Z$-range). We discuss this structure from front to back. Then, one first detects the forward shock (bow shock, at $Z \approx$ $170.8)$ that leads to pronounced increases in pressure and density, of a magnitude consistent with what the 1D problem demonstrated. Another discontinuity of a pure thermodynamic nature trails at $Z \approx 169.9$. In a zone $Z \in[169.6,169.9]$, we 
Tor: time=217.72
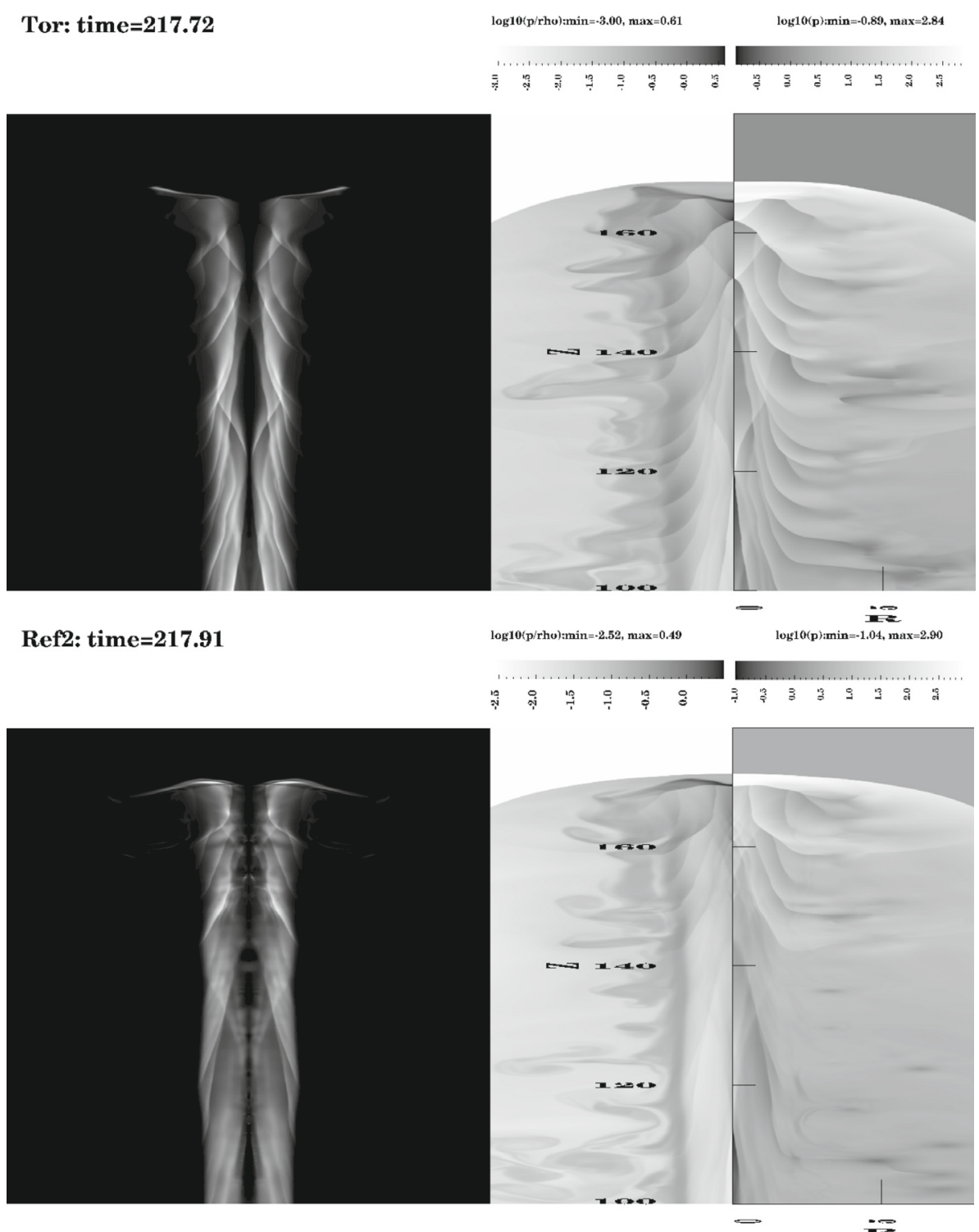

Pol: time=219.38

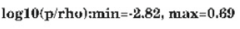

$\log 10(\mathrm{p}) \min =-0.55, \max =3.32$
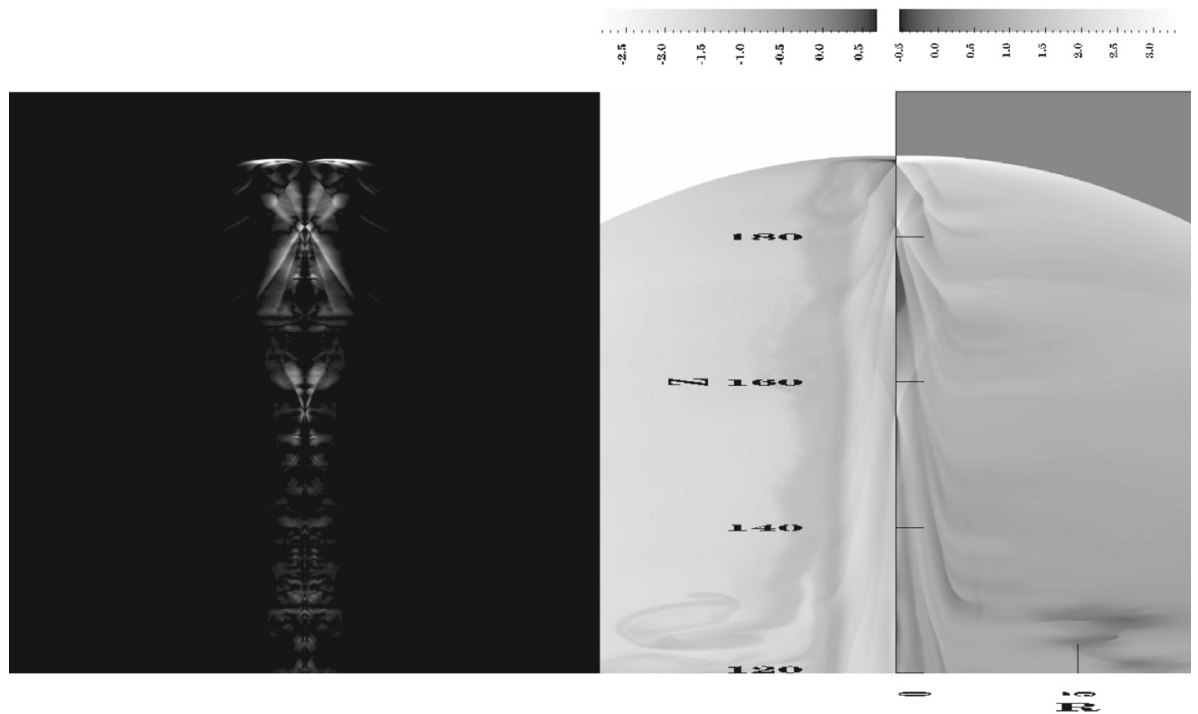

Fig. 10. For a sequence of models from the toroidal case (top), to a model with double the poloidal field magnitude as the reference case (middle), to nearly purely poloidal magnetic field (bottom): maps of arbitrarily scaled "power" given by $\Gamma^{2} v^{2} B^{2} \sin ^{2}(\Psi)$ (left), together with logarithmic greyscale plots of the temperature and pressure distributions. All plots correspond to instantaneous values at the end of the simulation, and only show a zoom in the top half of the entire domain. 

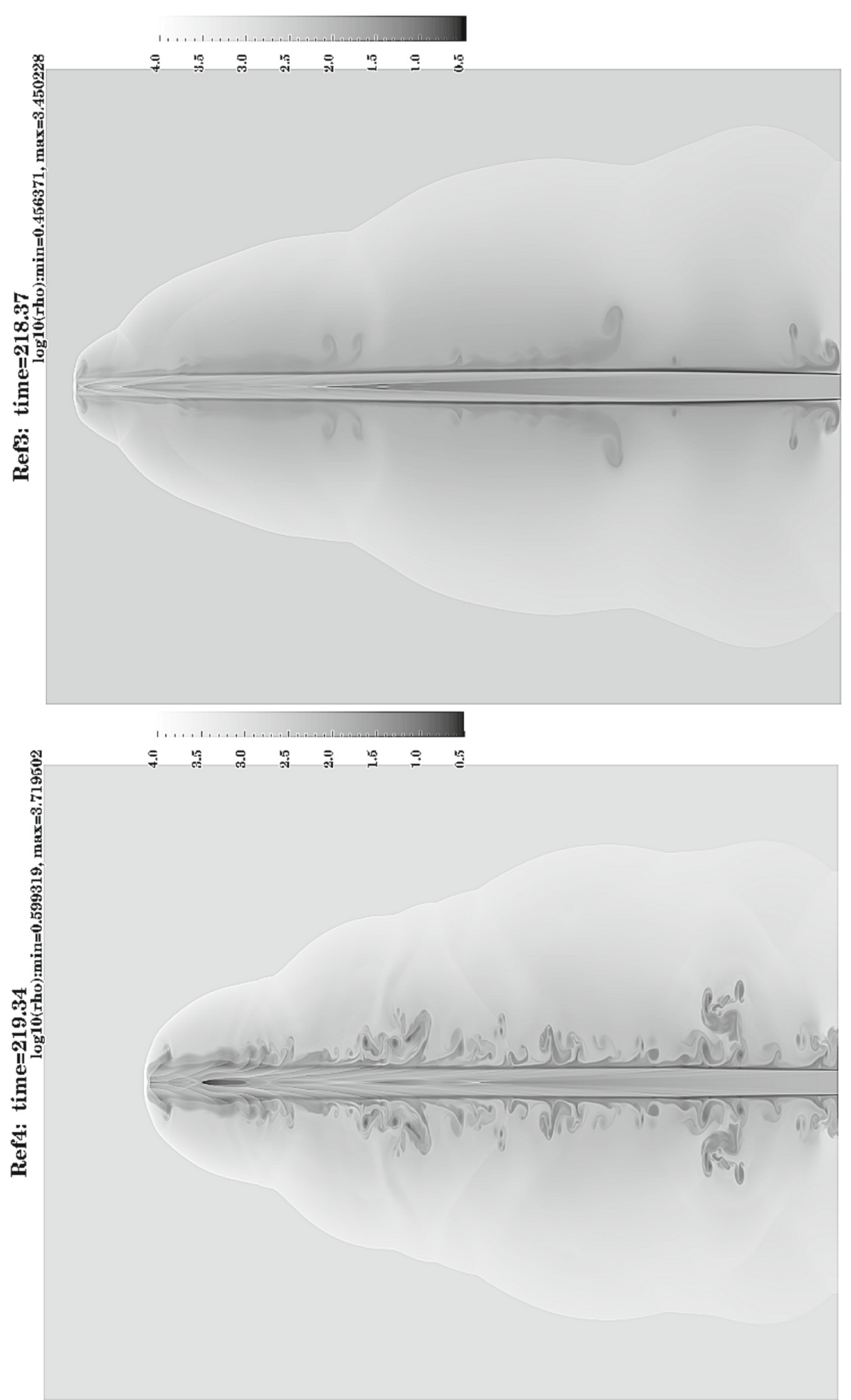

Fig. 11. For a heavier jet (top) versus a higher pressure jet (bottom): the proper density distribution at the end of the simulations. A reduced density contrast ensures fairly stable jet flows and little internal or cocoon structure. Increased jet pressure leads to more internal beam structure.

find the highest values for proper density $\rho \approx 6000$ and pressure $p \approx 1000$. Magnetic field variations all occur prior to $Z \approx 169.6$, where the density and pressure decrease from the values mentioned to values still significantly above their beam values. We can detect distinct variations in all three magnetic field components within the region $Z \in[168.8,169.6]$, coinciding with increased entropy $S=p \rho^{-\gamma}$ within this zone. At the trailing shock (Mach disk) located at $Z \approx 168.8$, the Lorentz factor drops from about 6 to 2 . The discontinuity seen at $Z \approx 162$ is the last internal shock prior to the jet head. There, as well as at the Mach disk, the azimuthal field is enhanced, in accordance with the increase in transverse magnetic field seen in the 1D problem from Fig. 3. The analogy cannot be expected to hold beyond such qualitative features: the actual 2D jet shows significant multi-D variation: the magnetic field nearly becomes purely radial and azimuthal within a thin layer $Z \in[169.3,169.9]$, and for $Z>169.9$ the uniform vertical cloud field of magnitude $B_{\mathrm{c}}=0.01$ is found. 
The sequence of models termed Pol, Ref1, Ref2, and Tor can be interpreted as a series with similar inlet characteristics in terms of average Lorentz factor and Mach number and by changing magnetic topology from nearly poloidal to pure toroidal. In accordance with the decreasing influence of the poloidal field in the jet beam (from Pol to Tor), the backflows tend to show a finer structure with more eddies being shed in the purely toroidal case. There is then more internal beam structure: multiple cross shocks are induced and they interact. Once more, full 3D simulations are needed to assess the generality of this result. The ringed vortex structures we obtain may break down and get stretched in a non-axisymmetric fashion. This can alter the overall field topology drastically and affect the backflow. We find that in all but the purely toroidal case, the regions of significant magnetic pressure are in the beam and backflows. The purely toroidal case carried a weak magnetic field, and this can be seen to trace the region occupied by shocked beam matter forming the inner cocoon.

As a qualitative means to addressing how different magnetic topologies lead to varying jet morphologies, we use this model sequence as follows. A formula quantifying the total emitted radiation by a single electron traveling at speed $\boldsymbol{v}$ in its relativistic cyclotron motion about a magnetic field $\boldsymbol{B}$ is given by (Rybicki \& Lightman 1979)

$P_{\text {tot }}=\frac{2 e^{4}}{12 \pi \epsilon_{0} m_{\mathrm{e}}^{2} c} v^{2} \Gamma^{2} B^{2} \sin ^{2}(\Psi)\left(\mathrm{J} \mathrm{s}^{-1}\right)$,

where $\Psi$ indicates the pitch angle between particle velocity $\boldsymbol{v}$ and magnetic field $\boldsymbol{B}$ (in Tesla). In our relativistic MHD simulations, we treat the plasma as a single fluid characterized by its bulk speed $\boldsymbol{v}$. A crude way to infer observational "synchrotron" intensity uses the bulk plasma speed in Eq. (22), together with detailed knowledge of the magnetic field distribution. In Fig. 10, the left panels produce arbitrarily scaled maps of the essential local dependence $v^{2} \Gamma^{2} B^{2} \sin ^{2}(\Psi)$ for the jet sequence, at the end of the simulations. It is to be noted that we present instantaneous local values of this "power map", which is not appropriate for quantifying true synchrotron emission for these relativistic jets, as we e.g. do not incorporate time-retardation effects. A procedure for incorporating all relevant relativistic effects uses the output of relativistic HD simulations and solves the relativistic radiative transfer equation by assuming optically thin conditions, as done in Komissarov \& Falle (1997). Recently, Zakamska et al. (2008) used analytic self-similar axisymmetric RMHD jet models to quantify both synchrotron emission and polarization maps by performing the proper integration along the line of sight in the observer's reference frame. In this paper, we restrict ourselves to quantifying the "power" dependence from Eq. (22), since for practical purposes, one would need to perform the data processing during the simulation, and make reasonable assumptions about the relativistic particle distribution function (which is not contained in the ideal RMHD simulation). The local instantaneous values give quantitative information on the overall flow and field topology, and it is this aspect that mostly concerns us here. Only the front half of the simulated domain is shown in Fig. 10, and a simultaneous quantification of the pressure and "temperature" $T=p / \rho$ is shown to the right. The highest pressure and temperature regions coincide with the (very narrow) regions between the Mach disk and the contact surface, and show up as bright regions ahead of the jet beams. Consistent with the higher pressure found in the frontal, compressed beam matter when more poloidal field is present, the associated (narrow) hot spot eventually dominates. In all cases, however, the complex shock interactions seen in the beam clearly show up. Given the aforementioned trend towards more complex shock interactions when more toroidal field structure is present, the power maps reflect this trend directly. The angle-dependent factor in formula (22), together with the magnetic field variation with radius, also explains the trend seen from more outer beam sensitivity in the almost toroidal case, to pronounced inner beam sensitivity at diagonal cross-shock fronts for more poloidal field configurations. This hints at the possibility of inferring field topology characteristics from true synchrotron emission maps, which need to be constructed rigorously from simulations such as the ones presented here.

\section{Thermodynamic variations}

The final two models are found in Fig. 11, showing the logarithm of their proper densities. The top panel treats the case with reduced density contrast, where the jet penetrates less dense material than in the reference case Ref1. The magnetized jet travels correspondingly faster, and a remarkably stable beam with little backflow features results. The stand-off distance to the first internal beam cross shock is also increased. In contrast, the Ref4 model that has a higher overall pressure compared to the reference case shows a similarly rich pattern of vortex structures. Since the magnetic field is identical in these models, the jet appearance seems to relate most dramatically on density differences, at least for these near-equipartition, kinetically dominated jets. Comparing the increased pressure jet case with the reference case in Fig. 6, the richly structured backflows are slightly more pronounced, and the internal beam density contrasts are more evident. Within the simulated time, the higher pressure jet propagation has had about one more phase of slight deceleration, reformation of the Mach disk, and consecutive acceleration shaping the overall bowshock. This is in accordance with its shorter internal sound crossing time. Quantitatively speaking, the highest proper density values (always found in the front of the jet head) are found for the reference case Ref1 where its instantaneous maximum is found $\rho_{\max }=6280$ (see Fig. 6), while the higher pressure case demonstrates values up to $\rho_{\max }=5236$. For the lower density contrast, we find a reduced value $\rho_{\max }=$ 2818 , as expected due to the lesser contrast and cloud density.

\section{Outlook}

We studied the morphology and propagation characteristics of a series of highly relativistic, helically magnetized jets. All were kinetic-energy dominated and, apart from the series of increasing averaged beam velocity, were similar in propagation speeds and overall magnetization (equipartition). With modest changes in magnetic topology, as well as in internal pressure and density, fairly distinct differences can be detected in the distribution of local power, as in the cocoon and internal beam structure. The radially stratified jets studied here show significant variation in the Lorentz factor across their diameter, and the high central "spine" (exceeding $\Gamma=20$ ) results in fairly elongated bow shocks. The magnetic helicity changes at internal cross-shocks act to repeatedly reaccelerate the jet. The lighter jets show much more of a fine structure in their backflows, and this will likely continue over higher density contrasts than those studied here. Previous studies (Leismann et al. 2005; Komissarov 1999) considered density ratios of 100 or more. The models here only considered density ratios of 5 to 10 and investigate jet propagation in less dense environments, which are known to lead to stabler 
jet configurations over longer distances. The sheet of the more poloidal field surrounding the jet beam proper, which we found in the simulations, also aids in stabilizing the beam.

In our simulations, the resulting jet propagation exhibits a small deceleration of the head of the (still) relativistic jet along its axis, only seen in the decrease in Lorentz factor along the axis occurring at the internal shocks. Such deceleration has been observed in various FR II jets, in correlation with an increase in the magnetic field intensity and particle density (Georgeanopoulos \& Kazanas 2004; Sambruna et al. 2006b). Tavecchio et al. (2006) show that this deceleration is compatible with the entrainment of the external gas by the jet. We plan to perform longer term simulations, in order to obtain a better description of the jet braking, as well as to study the impact of the external mass density compared to the jet one. In this kind of computation, we will explore regimes where the jet kinetic energy and the jet electromagnetic energy are of the same order of magnitude. The use of the AMR strategy will be a powerful tool in the study of the associated magnetic field and density amplification occurring near the jet head. We then aim to provide statements from modeling results about the ratio of the jet density to the external medium density, and use it to constrain the observational values better.

We also intend to explore the computationally challenging regime of Poynting-flux dominated jets with helical field topologies in future work. Since most AGN jets are likely to be associated with high Poynting-flux jets near the source, it will be interesting to study the transition from Poynting to kinetic energy dominated jets in even larger scale computations. Also, the stability of these jets in fully three-dimensional simulations is still unexplored. We demonstrated that the inlet jet magnetic topology for the sample studied here seems to be maintained over a significant distance, and the helicity measure showed only strong toroidal field concentrations in the localized vortices developing from the backflows. This was consistent with a trend followed from slower "non-relativistic" jets, where toroidal field gets created across the Mach disk and subsequently mixed into the cocoon. Since the toroidal field concentrations for the reference helical jet are mostly in localized, supersonically rotating vortices, their tendency to induce kink deformations may be less. Extremely high-resolution (grid-adaptive) 3D studies are called for to investigate this issue further.

Acknowledgements. Computations were performed on the K.U. Leuven Highperformance computing cluster VIC. Z.M. and R.K. acknowledge financial support from the Netherlands Organization for Scientific Research, NWO grant 614.000.421, and from the FWO, grant G.0277.08. These computations form part of the LMCC efforts.

\section{References}

Appl, S., \& Camenzind, M. 1988, A\&A, 206, 258

Appl, S., Lery, T., \& Baty, H. 1999, A\&A, 355, 818

Asada, K., Inoue, M., Uchida, Y., et al. 2002, PASJ, 54, L39

Baty, H., \& Keppens, R. 2002, ApJ, 580, 800

Begelman, M., \& Kirk, J. G. 1990, A\&A, 353, 66

Begelman, M. C., Blandford, R. D., \& Rees, M. J. 1984, Rev. Mod. Phys., 56, 255
Bergmans, J., Keppens, R., van Odyck, D. E. A., \& Achterberg, A. 2005, Lecture Notes in Computational Science and Engineering, 41, 223

Blandford, R. D., \& Znajek, R. L. 1977, MNRAS, 179, 433

Bodo, G., Rossi, P., Massaglia, S., et al. 1998, A\&A, 333, 1117

Bogovalov, S., \& Tsinganos, K. 2005, MNRAS, 357, 918

Carvalho, J. C., \& O’Dea, C. P. 2002, ApJS, 141, 337

Casse, F., \& Marcowith, A. 2005, Astropart. Phys., 23, 31

Celotti, A., Padovani, P., \& Ghisellini, G. 1997, MNRAS, 286, 415

Contopoulos, J. 1994, ApJ, 432, 508

del Zanna, L., Bucciantini, N., \& Londrillo, P. 2003, A\&A, 400, 397

Dulwich, F., Worrall, D. M., Birkinshaw, M., Padgett, C. A., \& Perlman, E. S. 2007, MNRAS, 374, 1216

Fendt, C. 1997, A\&A, 319, 1025

Gabuzda, D. C., Murray, É., \& Cronin, P. 2004, 351, L89

Georgeanopoulos, M., \& Kazanas, D. 2003, ApJ, 594, L27

Georgeanopoulos, M., \& Kazanas, D., 2004, ApJ, 604, L81

Ghisellini, G., Tavecchio, F., \& Chiaberge, M. 2005, A\&A, 432, 401

Giroletti, M., Giovannini, G., Feretti, L., et al. 2004, ApJ, 600, 127

Hardee, P. E. 2007, ApJ, 664, 26

Hardee, P. E., Walker, R. C., \& Gómez, J. L. 2005, ApJ, 620, 646

Harris, D. E., \& Krawczynski, H., 2006, ARA\&A, 44, 463

Jester, S., Harris, D. E., Marshall, H. L., \& Meisenheimer, K. 2006, ApJ, 648, 900

Kellermann, K. I., Lister, M. L., Homan, D. C., et al. 2004, ApJ, 609, 539

Keppens, R., \& Meliani, Z. 2007, Proceedings of ICCFD4 (Gent, Belgium, 2006)

Keppens, R., Nool, M., Tóth, G., \& Goedbloed, J. P. 2003, Comp. Phys. Comm., 153,317

Koide, S., Shibata, K., Kudoh, T., \& Meier, D. L. 2001, J. Korean Astron. Soc., $34,4,215$

Komissarov, S. S. 1999, MNRAS, 308, 1069

Komissarov, S. S., \& Falle, S. A. E. G. 1998, MNRAS, 288, 833

Leismann, T., Antón, L., Aloy, M. A., et al. 2005, A\&A, 436, 503

Li, Z.-Y., Chiueh, T., \& Begelman, M. C. 1992, ApJ, 394, 459

Lichnerowicz, A. 1967, Relativistic Hydrodynamics and Magnetohydrodynamics, (New York: Benjamin Press)

Majorama, A., \& Anile, A. M. 1987, Phys. Fluids, 30, 10

Martí, J. M., Müller, E., Font, J. A., Ibanez, J. M., \& Marquina, A. 1997, ApJ, 479,151

McKinney, J. 2006, MNRAS, 368, 1561

Meier, D. L. 2003, New Astro. Rev., 47, 667

Meliani, Z., Sauty, C., Vlahakis, N., Tsinganos, K., \& Trussoni, E. 2006, A\&A, 447,797

Meliani, Z., Keppens, R., Casse, F., \& Giannios, D. 2007, MNRAS, 376, 1189

Mignone, A., Massaglia, S., \& Bodo, G. 2005, Space Sc. Rev., 121, 21

Miller, K. A., \& Stone, J. M. 2000, ApJ, 534, 398

Mizuno, Y., Hardee, P., \& Nishikawa, K.-I. 2007, ApJ, 662, 835

O’Neill, S. M., Tregillis, I. L., Jones, T. W., \& Ryu, D. 2005, ApJ, 633, 717

Perucho, M., Lobanov, A., Martí, J. M., \& Hardee, P. E. 2006, A\&A, 456, 493

Rawlings, S., \& Saunders, R. 1991, Nature, 349, 138

Rybicki, G. B., \& Lightman, A. P. 1979, Radiative Processes in Astrophysics (New York: Wiley)

Sambruna, R. M., Gliozzi, M., Tavecchio, F., Maraschi, L., \& Foschini, L. 2006a, ApJ, 652, 146

Sambruna, R. M., Gliozzi, M., Donato, D., et al. 2006b, ApJ, 641, 717

Stawarz, L., \& Ostrowski, M. 2002, ApJ, 578, 763

Tavecchio, F., Maraschi, L., Sambruna, R. M., \& Urry, C. M. 2000, ApJ, 544, L23

Tavecchio, F., Maraschi, L., Sambruna, R. M., et al. 2004, ApJ, 614, 64

Tavecchio, F., Maraschi, L., Sambruna, R. M., et al. 2006, ApJ, 641, 732

Tóth, G., \& Odstrčil, D. 1996, J. Comp. Phys., 128, 82

van der Holst, B., \& Keppens, R. 2007, J. Comp. Phys., 226, 925

van der Holst, B., Keppens, R., \& Meliani, Z. 2008, Comp. Phys. Comm., submitted

Vlahakis, N., \& Königl, A. 2004, ApJ, 605, 656

van Putten, M. H. P. M. 1996, ApJ, 467, L57

Xu, C., Livio, M., \& Baum, S. 1999, AJ, 118, 1169

Zakamska, N. L., Begelman, M. C., \& Blandford, R. D. 2008

[arXiv: 0801.1120] 